\title{
Walls and Domain Shape Effects on the Thermal Marangoni Migration of Three-dimensional Droplets
}

\author{
Paolo Capobianchi, Marcello Lappa, Mónica S. N. Oliveira \\ James Weir Fluids Laboratory, Department of Mechanical and Aerospace Engineering, University \\ of Strathclyde, 75 Montrose Street, Glasgow, G1 1XJ, UK \\ email: paolo.capobianchi@strath.ac.uk, marcello.lappa@strath.ac.uk, \\ monica.oliveira@strath.ac.uk
}

The thermocapillary motion of liquid droplets in fluid media depends on a variety of influential factors, including the not yet fully understood role played by the presence of the walls and other geometrical constraints. In order to address this specific question, in the present work we rely on a rigorous mathematical and numerical framework (including an adaptive mesh strategy), which are key to perform physically consistent and computationally reliable simulations of such a problem given the different space scales it involves. Our final aim is the proper discernment of the triadic relationship established among viscous phenomena, thermal effects and other specific behaviour due to the proximity of the droplet to a solid boundary. Different geometric configurations are considered (e.g., straight, converging and diverging channels, droplets located near a single or adjacent walls) and distinct regimes are examined (including both ( $\mathrm{Ma}, \mathrm{Re}) \rightarrow 0$ and finite $\mathrm{Ma}$ flows). The results show that for straight channels the droplet generally undergoes a decrease in the migration velocity due to its proximity to the wall. Such a departure becomes larger as the Marangoni number is increased. In addition, a velocity component directed perpendicularly to the wall emerges. This effect tends to "pull" the droplet away from the solid boundary if adiabatic conditions are considered, whereas for thermally conducting sidewalls and relatively large values of the Marangoni number, the distortion of the temperature field in the region between the droplet and the wall results in a net force with a component directed towards the surface. For non-straight channels, the dynamics depend essentially on the balance between two counteracting factors, namely, the effective distribution of temperature established in the channel (for which we provide analytic solutions in the limit as $\mathrm{Re} \rightarrow 0$ ) and the "blockage effect" due to the non-parallel configuration of the walls. The relative importance of these mechanisms is found to change according to the specific regime considered (creeping flow or $\mathrm{Re}=O(1)$ ).

\section{Introduction}

The dynamics of gas bubbles and liquid droplets are prevalent in science and widely studied in many areas of engineering and materials processing (see, e.g., Lappa, ${ }^{1,2}$ Arienti and Sussman, ${ }^{3}$ Esmaeeli and Tryggvason ${ }^{4,5}$ ).

A drop or a bubble can move under the influence of driving forces of different origins. As an example, a drop of falling rain or a bubble rising in a denser liquid are put in motion because of the gravity force. ${ }^{6,7,8}$ The motion of liquid or gas inclusions in an external (matrix) fluid can also be induced (or "controlled" to a certain extent) by using other body forces such as those present in 
electric, magnetic and ultrasonic fields (see for instance, Behjatian and Esmaeeli, ${ }^{9}$ Nguyen et al., ${ }^{10}$ Brunet et al. ${ }^{11}$ ). Nevertheless, even in the absence of body forces, droplet or bubble displacement can be produced by another fundamental mechanism, the so-called thermal Marangoni (or thermocapillary) effect. This phenomenon, which requires a gradient of temperature to be present at the interface separating two fluid phases, becomes particularly important in all those circumstances where gravity is not the dominant effect, as attained in the microgravity environment provided by orbiting platforms, ${ }^{12,13}$ or when either the densities of the fluid pair are similar (see, e.g., Lappa et $a l . ;^{14,15}$ Lappa $^{16}$ and references therein) and/or the typical size of the dispersed phase is very small (e.g. atomised droplets, Grant et al., ${ }^{17}$ Arienti and Sussman ${ }^{3}$ ).

As a proof of its intrinsically multidisciplinary and complex nature, this problem has been addressed in the literature over the years by different research groups with very different interests and perspectives.

The first pioneering study on the thermocapillary migration of droplets dates back to Young et al. ${ }^{18}$, who, under some limiting assumptions (perfectly spherical drop of radius $R$ moving in an indefinite liquid under Stokes flow conditions) derived a landmark solution (in analytical form) of the governing equations. With such approach, velocity and temperature fields were considered to be fully established at every moment in time under the assumption of negligible inertia and convective effects. With this approximation the temperature field and the flow field decouple and this greatly simplifies the derivation of an analytical solution to the problem (it becomes possible to yield a precise relationship between the asymptotic (steady) droplet migration velocity and the properties of the considered fluids and the characteristics of the driving force).

After the initial study of Young et al., ${ }^{18}$ most ensuing works have been devoted to assessing the role played by mechanisms which were neglected in that initial work. As an example, Subramanian ${ }^{19}$ included the effect of convective transport of energy as a small perturbation (setting the Reynolds number to zero and using a perturbation expansion in the Marangoni number for $\mathrm{Ma}<1$ ); in practice, inertial terms were neglected in the momentum equation, whereas they were preserved in the energy equation (from a physical point of view this would be equivalent to considering a fluid with Prandtl number $\operatorname{Pr}>>1$, i.e. a liquid with a very high viscosity and a relatively small thermal diffusivity). Subramanian ${ }^{19}$ showed that in such conditions the migration speed of a drop can be reduced or enhanced with respect to the analytical solution by Young et al. ${ }^{18}$ depending on the values of the various parameters.

The opposite case, in which convective transport of heat is ignored (in the limit of $\mathrm{Ma} \rightarrow 0$ ) but small inertial effects are included in the momentum equation $(\operatorname{Re} \neq 0)$, was examined by Balasubramaniam and Chai, ${ }^{20}$ Haj-Hariri et $a l .{ }^{21}$ and Nadim et $a l .{ }^{22}$ still in the framework of perturbation techniques. In particular, Balasubramaniam and $\mathrm{Chai}^{20}$ extended the range of applicability of the solution of Young et al. ${ }^{18}$ under the constraint or requirement that the temperature field is in purely diffusive steady conditions (this occurs when $\operatorname{Pr}<<1$ and $\mathrm{Ma}=\operatorname{Re} \times \operatorname{Pr}$ remains small, i.e. $\mathrm{Ma}<1$ ). They also analysed the shape of the droplet in the limit of $\operatorname{Pr} \rightarrow 0$, illustrating that droplets of the same density as the matrix fluid do not deform at all, that less 
dense droplets and bubbles tend to deform oblately, and that droplets denser than the fluid matrix tend to elongate in the flow direction.

For similar conditions (convective transfer of heat neglected) and small but finite Re (i.e. influence of inertia taken into account), Haj-Hariri et al. ${ }^{21}$ and Nadim et al. ${ }^{22}$ calculated the correction to the migration velocity caused by the shape deformation. It was found that droplets with densities higher/lower than the outside liquid deform to prolate/oblate spheroidal shapes at small values of the capillary and Reynolds numbers. The corrections to the temperature field and the migration velocity of the droplet resulting from this deformation were obtained using the Lorentz reciprocal theorem. These authors illustrated that the migration velocity could increase, decrease, or remain unchanged according to the value of certain controlling parameters.

An analysis dealing with the asymptotic case of very high values of Re and Ma has been presented by Balasubramaniam and Subramanian ${ }^{23}$. They analysed the problem relating to the steady Marangoni migration of a spherical drop in a continuous phase under the idealised conditions $(\mathrm{Ma} \rightarrow \infty$ and $\mathrm{Re} \rightarrow \infty)$, assuming that inertial terms in the momentum equation and convectivetransport terms in the energy equation dominate over the corresponding molecular-transport terms. In such a mathematical context (partially based on the earlier model by Harper and Moore ${ }^{24}$ ), the migration velocity of the drop was obtained on the basis of a potential-flow theory, where the rate at which work is done by the thermocapillary stress was equated to the rate of viscous dissipation of energy; the method of matched asymptotic expansions was also employed to solve the conjugate heat-transfer problem in the two phases (characterised by the presence of thin thermal boundary layers both outside and within the drop). In physical terms it was found that in the limit as $\mathrm{Ma} \rightarrow \infty$ the velocity of a drop becomes proportional to the square of the temperature gradient and the cube of the radius of the drop.

More recently (essentially over the last two decades), the availability of powerful computers (including the possibility to resort to multi-processor computations) and the development of modern moving-boundary methods, such as the Volume of Fluid (VOF) or the level set (LS) techniques (see, e.g., Haj-Hariri et al.; ${ }^{25}$ Rudman; ${ }^{26}$ Gueyffier et al.; ${ }^{27}$ Sussman and Fatemi; ${ }^{28}$ Sussman and Puckett $^{29}$; Tryggvason et al. ${ }^{30}$ just to mention some initial efforts), has made possible addressing the problem directly in the framework of direct numerical discretisation and solution of the governing balance equations in the most general (time-dependent and non-linear) form. Along these lines, for instance, it is worth mentioning the works of Yin et al., ${ }^{31,32}$ Zhao et al., ${ }^{33}$ Brady et al., ${ }^{34}$ Balcázar et $\mathrm{al}^{35}$, who performed parametric studies to examine the influence of typical non-dimensional numbers on the migrating process of both rigid and deformable drops.

As a common finding, all these studies for finite values of Re and Ma highlighted that when convective transport is important the internal circulation in the drop has a profound influence on the temperature distribution in its vicinity and hence on its migration speed. Moreover, thermal boundary layers can form in front of the droplet, and fluid-dynamic instabilities can even develop, when the value of the Marangoni number is sufficiently large. 
In addition to the above mentioned strategies, it is also worth mentioning the recent development of lattice Boltzmann methods (LBM, ( Liu et $a l^{36}$, Liu and Zhang ${ }^{37}$ ). Other techniques based on the phase-field method have been also demonstrated to be suitable for the simulation of the thermocapillary migration of droplets. In such a context, is worth mentioning the work of Guo and $\mathrm{Lin}^{38}$ who have adopted the phase field method in conjunction with a novel approach based on a "non-classical" energy balance equation. Liu and Valocchi ${ }^{39}$ combined the capabilities of the lattice Boltzmann and phase field methods showing that such strategy can be successfully adopted for the simulation of thermocapillary flows. Similar approaches have been also used to study the motion of droplets under the influence of localised sources of heat (Liu et $a l^{40,41}$ ). Although much progress has been made on the modelling and understanding of such phenomena, a specific gap has still to be bridged with respect to another of the important aspects embedded in the aforementioned IBVP problem (which elegantly encapsulates all the facets of this subject), namely the role played by the presence of solid surfaces (walls) and the specific three-dimensional shape (geometry) of the considered container.

Apart from the general interest from the point of view of applied mathematics, which attaches to such extensions, wall-effects and geometrical constraints (see e.g. Meyyappan et al. ${ }^{42}$ ) in general may be an important source of observed discrepancies between idealised numerical simulations (carried out under the assumption that the droplet motion occupies exactly the "centre" of the container, e.g., the symmetry axis for a cylindrical container) and the results provided by effective experiments (recently made possible by the availability of microgravity platforms such as the ISS (see, e.g., Wozniak, ${ }^{43}$ Balasubramaniam et al. ${ }^{44}$ and Hadland et $a l .{ }^{12}$ ); these experiments have provided disjoint glimpses of a range of qualitatively and quantitatively different results in widely different parts of the parameter space.

In order to address this specific question, in the present work we rely on non-dimensional problem formulation and numerical simulation based on an adaptive mesh strategy able to capture in a single numerical treatment both localised and large-scale effects, which are key to the physically consistent and computationally reliable problem simulation.

Our final aim is the proper discernment of the non-trivial relationship established among different effects, including: the relative importance of surface-tension forces and viscous forces (as measured by the Marangoni number), the proximity of the droplet to a solid wall, and the shape of the container itself. Different geometric configurations are considered along these lines (e.g., straight, converging and diverging channels, droplets located near a side wall or near the intersection of perpendicular walls, i.e. corners). The analysis also progresses through the examination of distinct regimes (including both creeping and large-Ma flows).

\section{Mathematical model and Numerical Approach}

\section{A. Governing equations and boundary conditions}


The droplet undergoing Marangoni migration is assumed to be surrounded by an immiscible liquid subjected to a constant temperature gradient $\nabla_{\infty} T$. Classically, this problem can be treated by introducing two distinct phases, each with its set of balance equations, and appropriate conditions at the interface to guarantee inter-phase coupling (see, e.g., Tryggvason et al. ${ }^{45}$ ). However, an alternate and more effective approach can be based on "interface capturing methods", such as the Level-Set (LS) or the Volume of Fluid (VOF). These techniques rely on a different strategy generally referred to as "single-fluid" or "one-fluid" approach (see, e.g., Lappa ${ }^{46}$ and references therein). Such alternate strategy is based on considering the system as if composed by one single phase with non-constant properties (undergoing discontinuities across the fluid-fluid boundaries). With regard to the balance of momentum, the presence of the interfacial stresses is accounted for adding "extra" forces to the related equation.

Assuming that the effect of gravity and other external body forces are negligible, the conservation of momentum is cast in condensed form as

$$
\rho\left(\frac{\partial \mathbf{u}}{\partial t}+\mathbf{u} \cdot \nabla \mathbf{u}\right)=-\nabla p+\nabla \cdot\left[\mu\left(\nabla \mathbf{u}+(\nabla \mathbf{u})^{\mathbf{T}}\right)\right]+\mathbf{f}_{\sigma}
$$

where $t$ is the time, $\rho$ and $\mu$ are the fluid density and viscosity, respectively, $p$ is the pressure and $\mathbf{u}$ the velocity vector. The last term $\mathbf{f}_{\sigma}$ is a force accounting for the capillary $\left(\mathbf{f}_{\sigma, \mathbf{n}}\right)$ and thermocapillary $\left(\mathbf{f}_{\sigma, \tau}\right)$ forces at the interface:

$$
\mathbf{f}_{\sigma}=\mathbf{f}_{\sigma, \mathbf{n}}+\mathbf{f}_{\sigma, \tau}=\sigma_{0} k \mathbf{n} \delta_{S}+\nabla_{\|}-(\boldsymbol{r}) \delta_{S}
$$

Moreover, $k$ and $\mathbf{n}$ are the interface curvature and the related normal unit vector, respectively; $\mathbf{I}$ is

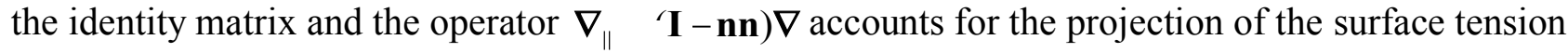
gradient along the direction tangent to the interface. The term $\delta_{S}$ represents a function which takes a value of 1 at the interface and is zero elsewhere. Since the interfacial tension, $\sigma$, is a function of the temperature, $T$, the related dependence is expressly included in Eq. 2. The complete mathematical model also requires consideration of the continuity equation for an incompressible flow (Eq. 3) and the energy transport equation (Eq. 4):

$\nabla \cdot \mathbf{u}=0$

$\rho c_{p}\left(\frac{\partial \mathbf{u}}{\partial t}+\mathbf{u} \cdot \nabla T\right)=\nabla \cdot(\kappa \nabla T)$

where $c_{p}$ is the specific heat and $\kappa$ the thermal conductivity coefficient of the fluid. Following common practice for this kind of problems (see, e.g., Yin ${ }^{32}$ ), all material properties are assumed to be constant in each phase and are evaluated at a suitable reference temperature. The dependence on temperature, however, is retained for the surface tension $\sigma$ via a linear relationship: 
$\sigma(T)=\sigma_{0}+\sigma_{T}\left(T-T_{0}\right)$

where $\sigma_{0}$ is the interfacial tension at the reference temperature $T_{0}$, and $\sigma_{T}=\partial \sigma / \partial T$ is negative for most known fluids (see e.g. Balasubramanian and Subramanian ${ }^{47}$ ).

\section{B. The Simplified LS-VOF Method}

Our solver is based on a coupled LS-VOF approach (relying on the hybrid formulation originally elaborated by Albadawi et al., ${ }^{48}$ see also Sussman and Puckett ${ }^{29}$ ) implemented into the OpenFOAM platform $^{49}$ as an extension of the standard VOF solver "interFoam". The simplified coupled LSVOF for an isothermal system is based on the solution of Eqs. (6)-(10). The equation for the volume fraction reads:

$$
\frac{\partial \alpha}{\partial t}+\nabla \cdot(\alpha \mathbf{u})+\nabla \cdot\left(\alpha(1-\alpha) \mathbf{u}_{c}\right)=0
$$

where $\alpha$ is the volume fraction ( $\alpha=1$ in the drop, $\alpha=0$ in the matrix fluid, and $0<\alpha<1$ at the interface) and $\mathbf{u}_{c}$ is an artificial "compressive velocity" ${ }^{50}$ required to damp the numerical diffusion at the interface (note that in the solver there is no explicit reconstruction of the interface). Although this coupled methodology produced significant observable improvements in the results of our simulations (in terms of accuracy and mitigation of the so-called "parasite" currents with respect to the original two-phase solver), we had to implement some additional countermeasures required to improve "algorithm stability", especially with respect to the interface "behaviour" (where Marangoni stresses of thermal nature are produced). This was accomplished by "proper" smoothing of both the level set and the volume of fluid phase functions, as further described in Sect. IIC.

The resulting time-marching procedure can be sketched as follows: in order to calculate the level set function $\varphi$, initially the field $\varphi_{0}=(2 \alpha-1) \Gamma$ is determined, where $\Gamma=0.75 \Delta x$ and $\Delta x$ is the grid resolution (see Albadawi at al. ${ }^{48}$ ). Subsequently, we solve a re-initialisation equation (see, e.g., Sussman and Fatemi ${ }^{28}$ ) with the initial condition $\varphi(\mathbf{x}, 0)=\varphi_{0}(\mathbf{x})$ :

$$
\frac{\partial \varphi}{\partial \tau}=\operatorname{Sgn}\left(\varphi_{0}\right)(1-|\nabla \varphi|)
$$

where the term $\operatorname{Sgn}\left(\varphi_{0}\right)=\varphi_{0} /\left|\varphi_{0}\right|$ represents the sign function. Once the scalar field $\varphi$ is known at each point, it is possible to evaluate the curvature at the interface

$$
k(\varphi)=-\nabla \cdot \mathbf{n}(\varphi)
$$


with $\mathbf{n}(\varphi)=\nabla \varphi /|\nabla \varphi|$ being the unit vector perpendicular to the interface. Finally, the term accounting for the capillary and thermocapillary forces at the interface described by Eq. 2 is evaluated, leading to cast the momentum equation in compact form as

$\rho\left(\frac{\partial \mathbf{u}}{\partial t}+\mathbf{u} \cdot \nabla \mathbf{u}\right)=-\nabla p+\nabla \cdot\left[\mu\left(\nabla \mathbf{u}+(\nabla \mathbf{u})^{\mathbf{T}}\right)\right]+\sigma k(\varphi) \eta(\varphi) \nabla \varphi+\sigma_{T} \nabla_{\|}^{\boldsymbol{T}^{\prime}} \boldsymbol{\nabla} \nabla \alpha \mid$

where

$\eta(\varphi)= \begin{cases}0 & \text { if }|\varphi|>\varepsilon \\ \frac{1}{2 \varepsilon}\left(1+\cos \left(\frac{\pi \varphi}{\varepsilon}\right)\right) & \text { if }|\varphi| \leq \varepsilon\end{cases}$

and

$2 \varepsilon=3 \Delta x$

The reader is referred to the review by Lappa ${ }^{46}$ for additional information about the mathematical manipulations required to turn the surface force in Eqs. 1-2 into a corresponding volume force spread over a region of finite thickness, which no longer relies on the use of the impulse $\delta$ function. Additional details on the dependence on $\varphi$ and $\alpha$ present in Eq. 9 are provided in Sect. II C.

\section{Implementation of the thermal Marangoni migration method in OpenFOAM}

The solution strategy has been based on a classical Finite Volume Method (FVM) approach relying on the governing equations cast in integral form over a set of control volumes. More precisely, the equations have been solved in a Cartesian coordinate system using a three-dimensional mesh composed of hexahedrons with the open-source tool-box OpenFOAM® (see Capobianchi et $a l^{51}$ and references therein).

With OpenFOAM, as for all classical techniques pertaining to the so-called category of fractional step methods (also known under several other names, such as projection methods or pressure-based methods), the velocity and pressure fields are determined in a disjoint (sequential) manner. In particular, the pressure is computed via the solution of a Poisson-like equation obtained combining the discrete momentum equation and the continuity equation (the so-called PISO algorithm, see, e.g., Jang et $a l .{ }^{52}$ or the exhaustive book by Moukalled et al. $^{53}$ for additional details). The implementation of the PISO method in OpenFOAM relies on a non-staggered collocation of the different problem variables on the underlying computational grid (which means all primitive variables are located at the same grid points); in order to prevent the code from developing spurious oscillations (caused by a not well-resolved coupling between pressure and velocity, see, e.g., Choi 
et $a l^{54,55}$ and references therein), the convective flux at each control volume face is determined resorting to the scheme originally developed for Cartesian grids by Rhie and Chow ${ }^{56}$, which in OpenFOAM has been extended and adapted for generalised coordinates.

All the convective and diffusive terms are treated implicitly whereas other source terms eventually present in the equations (i.e. the surface-tension term in the momentum equation) are discretised explicitly.

The solution of the energy equation has been implemented in the classical segregated ${ }^{57,58}$ ), i.e. the momentum and energy equations are solved one at a time, with the coupling implemented in an explicit way.

For all the cases, the first order accurate implicit Euler temporal scheme has been used. The diffusive terms have been discretised using a standard central difference scheme, while in the momentum and energy equations the convective terms have been discretised using the QUICK scheme. Such choices proved to be the best compromise in terms of 1) algorithm stability, 2) mesh convergence, and 3) numerical accuracy (the reader is referred to the grid-refinement tests and the validation studies reported in Sect. III A1).

In addition to the above careful treatment, we had to use properly mollified variables to increase algorithm stability and mitigate unphysical effects at the interface. More precisely, the smoothing was applied to each "relevant variable" $\chi$ (representing various variables required by the LS and VOF implementation in different parts of the solver, as needed) using a "pure diffusive" evolution equation $\chi_{m o l}^{n+1}=\chi_{m o l}^{n}+\left(\nabla^{2} \chi_{m o l}^{n}\right) \Delta \tau$ where $\tau$ is an artificial or fictitious time, to be solved with the initial condition for a prefixed number of cycles $n$ (the condition $n=0$ corresponding to the recovery of the original non-smoothed function). We used mollified quantities to evaluate the new curvature at each time step, i.e.

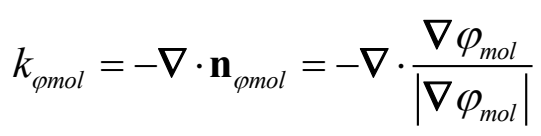

where $\varphi_{m o l}$ is the smoothed version of $\varphi$.

As discussed in Sect. II A, accounting for surface-tension effects requires two additional source terms in the momentum equation (see Eq. 2). On the basis of an optimisation strategy based on a trial-and-error approach, we could obtain the best results using the mollified level set function to determine the unit vector perpendicular to the interface, while retaining a non-mollified volume fraction in the gradient appearing in the expression of the thermal contribution (see Eq. 13). The level set function was also used accordingly to determine the curvature.

$$
\mathbf{f}_{\sigma, \boldsymbol{\tau}}=\sigma_{T} \nabla_{\|}{ }^{\top /} \nabla \chi\left|=\sigma_{T}\left(\mathbf{I}-\mathbf{n}_{\varphi m o l} \mathbf{n}_{\varphi m o l}\right) \nabla T\right| \nabla \alpha \mid
$$

Following common practice in the literature, ${ }^{59}$ the smoothing philosophy has also been applied to the fluid properties (assumed to be constant in each phase) in order to prevent the algorithm from 
developing spurious oscillations due to the discontinuity established at the liquid-liquid interface. In our hybrid implementation we decided to rely on a standard VOF approach expressing each property at the interface as

$$
\gamma=\alpha_{m o l} \gamma_{1}+\left(1-\alpha_{m o l}\right) \gamma_{2}
$$

In order to improve the accuracy without increasing too much the computational cost (the considered problem is $3 \mathrm{D}$ ), we also deemed it necessary to use an adaptive mesh refinement technique (especially for relatively high values of the Marangoni number for which relatively thin thermal boundary layers tend to emerge at the matrix-droplet interface, as further explained in Sect. III). The approach implemented in OpenFOAM is based on the h-refinement strategy, in which additional computational points are inserted locally in some regions without disturbing the rest of the mesh. It is also possible to remove points from regions in which they are no longer necessary through an "unrefinement procedure". To define the regions to be enriched with additional points we have used as a controlling variable, a mollified scalar field, $\alpha_{\text {mol }}^{*}$, expressly created for such a purpose and defined using the volume fraction $\alpha$ (with points being dynamically added to the region where $\alpha_{\text {mol }}^{*}>\alpha_{\text {treshold }}^{*}$ and removed from the rest of the domain). The method is particularly suitable for problems with discontinuous properties such as those considered here. ${ }^{60-62}$

Before the validation and discussion of the results in the next section, here we list the independent non-dimensional parameters governing the physics of the flow under discussion. These are: the fluid properties ratios $\tilde{i} \quad, \tilde{r} \quad, \tilde{r}_{i} \quad z_{p, m}$ and $\tilde{r}$, the capillary number $\mathrm{Ca}=\sigma_{T}\left(\nabla_{\infty} T\right) R / \sigma_{0}$, the Marangoni number $\mathrm{Ma}=\sigma_{T}\left(\nabla_{\infty} T\right) R^{2} / \alpha_{m} \mu_{m}$ and either the Reynolds number $\operatorname{Re}=\rho_{m} \sigma_{T}\left(\nabla_{\infty} T\right) R^{2} / \mu_{m}^{2}$ or the Prandtl number $\operatorname{Pr}=\mu_{m} / \rho_{m} \alpha_{m}$, (since $\left.\mathrm{Ma}=\operatorname{Re} \cdot \operatorname{Pr}\right)$. The subscripts " $m$ " and " $d$ " denote matrix and drop, respectively. The above mentioned parameters can be formally introduced by putting the governing equations (Eqs. 1, 3, 4) in non-dimensional form using $t_{r e f}=\mu_{m} / \sigma_{T} \nabla_{\infty} T$ as a reference time, $L_{r e f}=R$ as a length reference scale, and $U_{\text {ref }}=\sigma_{T} R \nabla_{\infty} T / \mu$ as a velocity scale.

\section{Numerical Results}

\section{A. Marangoni drop migration in $3 D$ geometries with uniform square cross-section}

\section{Reproducing available experimental results at finite (moderate) values of Re and Ma}

The performance of our solver has been already assessed in the case of thermocapillary migration of droplets under creeping flow conditions, i.e. in the limiting case of vanishing Marangoni and Reynolds numbers for which the velocity can be expressed via the so-called Young formula, ( $c f$. Eq. 
(15), the reader being referred to Capobianchi et al.$^{51}$ for additional details, which are not duplicated here for the sake of brevity). In this section, we focus on the case where thermal and momentum convective terms play a key role, i.e. finite values of the Marangoni and Reynolds numbers.

$$
U_{Y G B}=\frac{2\left|\sigma_{T}\right|\left(\nabla_{\infty} T\right) R / \mu_{m}}{\left(2+\frac{k_{d}}{k_{m}}\right)\left(2+3 \frac{\mu_{d}}{\mu_{m}}\right)}
$$

In particular, the reliability and accuracy of the code are tested considering the experimental measurements of Hadland et al. ${ }^{12}$ obtained in microgravity conditions (during a NASA space shuttle mission), in which the thermal Marangoni migration of a fluorinert drop enclosed in a box filled with silicone oil was examined. The experiments were conducted in a domain with a square crosssection $(4.5 \times 4.5) \mathrm{cm}^{2}$ and a height of $6 \mathrm{~cm}$. Droplets of different diameter were considered so that a relatively large range of Marangoni numbers was covered (from approximately 10 to 4000).

An imposed temperature gradient $\nabla_{\infty} T$ was applied by maintaining two opposite sides of the domain at a different temperature (as shown in Fig. 1). As the droplet moved from the cold region to the hot region, its position was monitored and recorded using a motion picture camera.

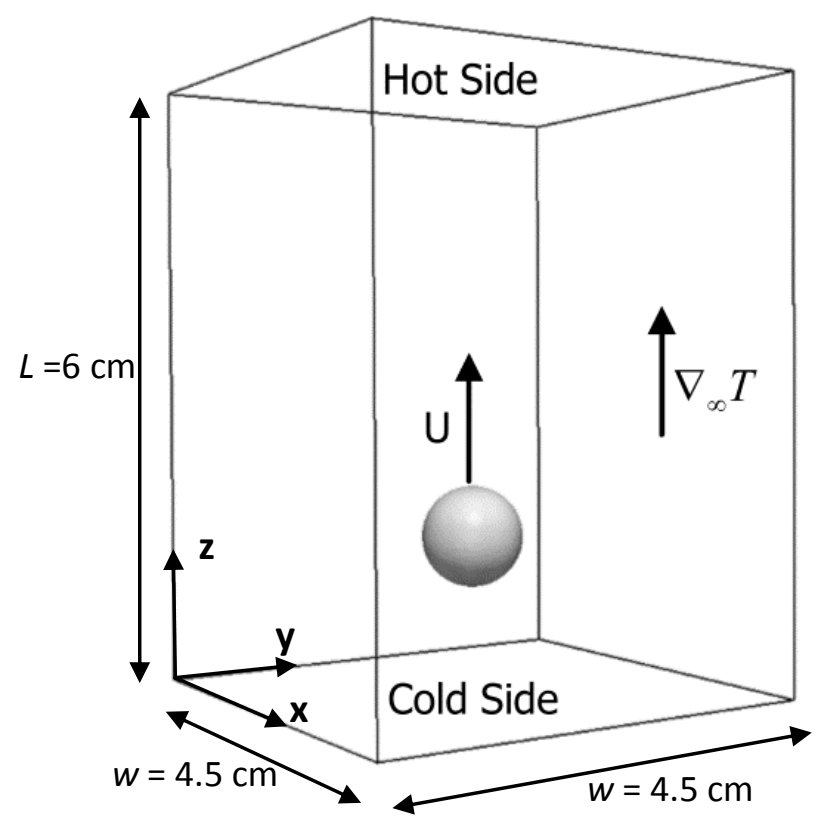

FIG. 1: Schematics of the system (equivalent to the experiment of Hadland et al. ${ }^{12}$ ) and coordinate axes considered in the numerical study.

Here, the same domain is reproduced numerically assuming fluid properties corresponding to the average system temperature as shown in Table I. As boundary conditions for the temperature we use fixed (constant) temperature on the end walls and adiabatic (results in Sects. 3.A.1, 3.A.2, 3.A.3 and 3.B) or conducting (results in Sects. 3.A.4) conditions for the side-walls, while for the velocity we set "no-slip" conditions at each wall. As for initial conditions, at the beginning of the calculation the whole flow field was considered at rest whilst, in terms of temperature, we imposed a uniform 
linear distribution. While such condition on the temperature can have an impact on the initial (transient), the velocity displayed by the droplet when steady conditions are attained does not depend on them (see e.g. Brady et al. ${ }^{34}$ )

Table I Fluid properties adopted for the simulations (density and viscosity have been calculated at the average temperature of $313 \mathrm{~K}$ using the correlations available in Hadland et al. ${ }^{12}$ )

\begin{tabular}{cccccc}
\hline & $\begin{array}{c}\text { Density } \\
{\left[\mathrm{kg} / \mathrm{m}^{3}\right]}\end{array}$ & $\begin{array}{c}\text { Viscosity } \\
{[\mathrm{Pa} \mathrm{s}]}\end{array}$ & $\begin{array}{c}\text { Thermal } \\
\text { conductivity } \\
{[\mathrm{W} / \mathrm{m} \mathrm{K}]}\end{array}$ & $\begin{array}{c}\text { Specific } \\
\text { heat } \\
{[\mathrm{J} / \mathrm{kg} \mathrm{K}]}\end{array}$ & $\begin{array}{c}\text { Prandtl } \\
\text { Number } \\
{[-]}\end{array}$ \\
\hline Matrix & 918.3 & 0.00729 & 0.1339 & 1778 & 98.86 \\
Droplet & 1728 & 0.00102 & 0.0630 & 1047 & 17.03 \\
\hline
\end{tabular}

Moreover, a constant value of the capillary number is considered ( $\mathrm{Ca}=0.2$ in all cases). This value is sufficiently small (although not corresponding exactly to the experimental value) to guarantee non-appreciable deformation of the droplet as in the experiments and, simultaneously sufficiently high to prevent the development of significant spurious currents in the numerical simulation (see e.g., the arguments given in this regard by Zhao et al. ${ }^{33}$ ).

Our goal is to test the solver considering effective conditions, i.e. adopting the same geometrical constraints and flow conditions of a real experiment and performing fully three-dimensional (3D) simulations (most of past results available in the literature have been obtained under the approximation of axisymmetric flow).

As outlined in the introduction, to discretize the domain we have used the adaptive mesh capabilities offered by the OpenFOAM platform in order to ensure a sufficiently refined mesh in the region of the droplet, where a better resolution is required (the refinement being applied essentially to the whole drop and its surrounding area). In order to assess the sensitivity of the solution to the mesh density, we conducted a set of simulations considering three different levels of refinement (indicated with $\mathrm{M}_{1}, \mathrm{M}_{2}$ and $\mathrm{M}_{3}$ ) obtained by halving the mesh spacing at each refinement and a fairly high value of the Marangoni number $(\mathrm{Ma}=100)$ as a representative reference condition. We selected the time step on the basis of stability considerations related to the need to control the level of the unphysical spurious velocities at the interface (Galusinski and Vigneaux ${ }^{63}$ ). The time step constraint is such that

$\Delta t \leq 0.5\left(C_{2} \frac{\mu \Delta x}{\sigma}+\sqrt{C_{2} \frac{\mu \Delta x}{\sigma}+4 C_{1} \frac{\rho \Delta x^{3}}{\sigma}}\right)$

where $C_{1}$ and $C_{2}$ are coefficients that depend on the particular solver used (as indicated by Deshpande et al. $^{64}$ ). 
Table II: Characteristics of the meshes used for the mesh-independence assessment study

\begin{tabular}{lccc}
\hline & Mesh $\mathrm{M}_{1}$ & Mesh M & Mesh M $_{3}$ \\
\hline Nr of cells per drop diameter & 28 & 56 & 112 \\
Grid resolution $(\Delta x=\Delta y=\Delta z)$ & 0.0003572 & 0.0001786 & 0.0000893 \\
\hline
\end{tabular}
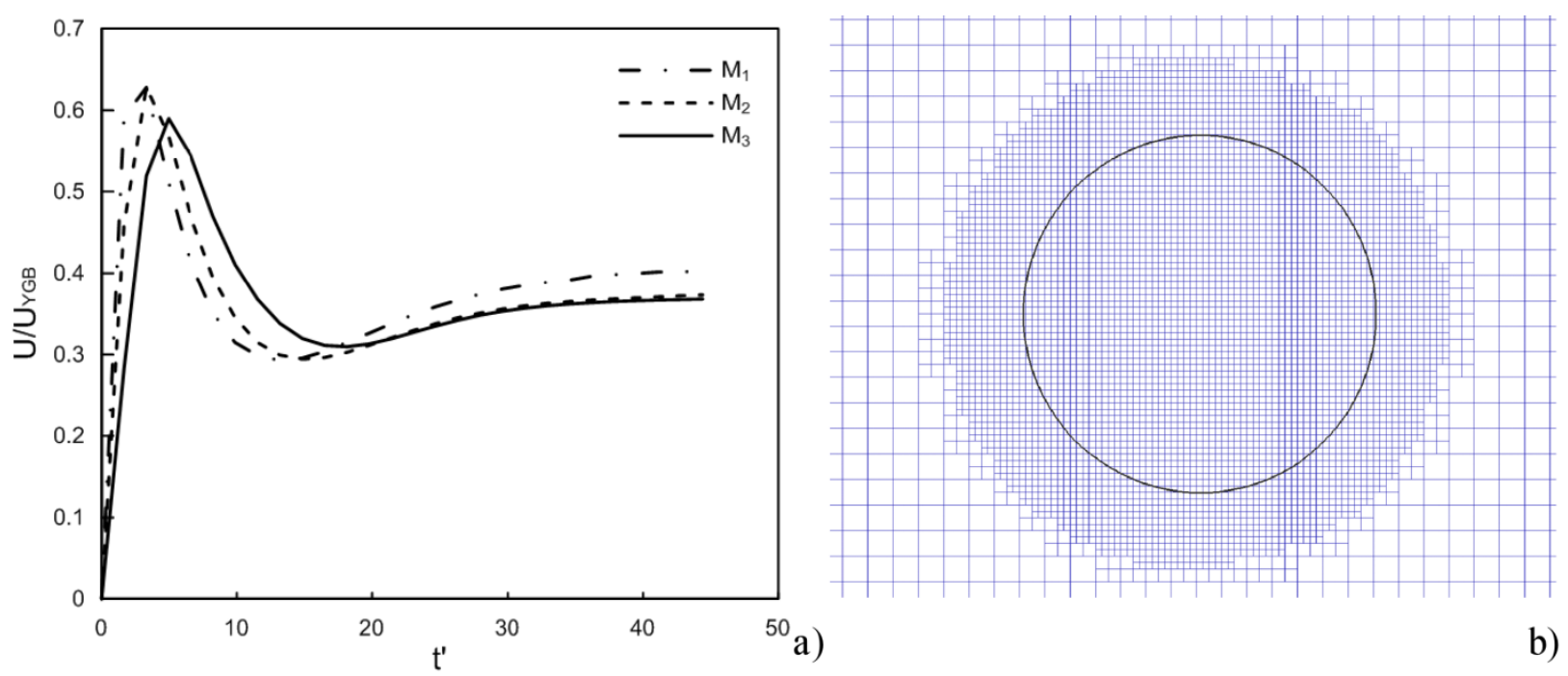

FIG. 2: a) Outcomes of the 3D mesh refinement study for $\mathrm{Ma}=100$ (scaled droplet velocity for three different uniform mesh resolution); b) Adaptively refined 3D mesh with "local" resolution $\mathrm{M}_{2}$ in a plane parallel to the plane $x=0$ passing through the centre of the droplet.

On the basis of the results provided by the mesh independence study summarised in Fig. 2a, we could discern the minimum resolution needed to obtain grid-independent results (the resolution named " $\mathrm{M}_{2}$ " in Table II). Note that, while mesh $\mathrm{M}_{1}$ is not resolved enough to capture the thermal boundary layer established in the front region of the droplet (we performed this study considering $\mathrm{Ma}=100$, thus a thermal boundary layer is expected to be established in the front region of the droplet), meshes $\mathrm{M}_{2}$ and $\mathrm{M}_{3}$ capture the physics correctly, as shown by the migration velocity results in Fig. 2a, which converge to very similar values of the final velocity (percentage difference for the final velocity smaller than $1 \%$ ). It is worth pointing out that a uniform $3 \mathrm{D}$ mesh having the same resolution throughout the domain would have required $\sim 26$ millions of cells, which has to be regarded as an almost "prohibitive task" in the context of a parametric analysis such as that conducted in the present work. Using the adaptive mesh strategy described above, the simulations reported in the present study took a total of approximately three months of effective CPU time (each simulation requiring on average $48-72$ hours).

We assumed the droplet to be initially in quiescent conditions close to the bottom cold side as shown in Fig. 1 (the centre of the drop was located at $(2.25,2.25,1.5) \mathrm{cm}$ ) with a temperature equal to that of the surrounding fluid. As pointed out by Brady et al. ${ }^{34}$ this assumption might be nonrealistic and could have an influence on the initial transient; however, it has no impact on the terminal velocity and, therefore, such simplification does not constitute an issue for our purposes. 
Figure 3 shows the drop migration velocity normalised with the theoretical velocity predicted by the model of Young et al. ${ }^{18}\left(U_{Y G B}\right.$ reported in Eq. (15)) as a function of the dimensionless time. A range of different values of the Marangoni number (from 2 up to 500) has been considered.

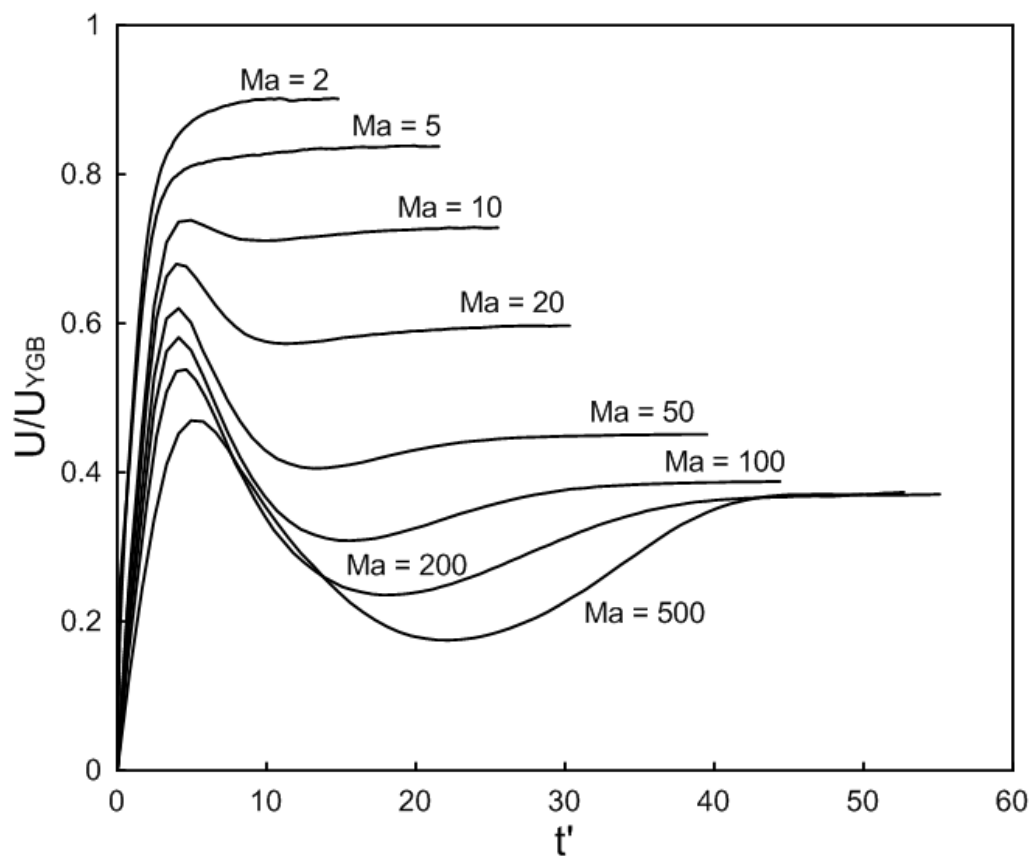

FIG. 3: Time evolution of the normalised droplet velocity for different values of the Marangoni number

For Ma up to 100 , in agreement with previous simulations (see e.g. Yin et al. ${ }^{32}$ ) our results have confirmed that after an initial transient time the droplet velocity reaches a plateau region, attaining a steady state. For the highest values of Ma considered here $(\mathrm{Ma}=200$ and 500), however, the size of the considered domain was not sufficiently long to allow the droplet to reach such a state. As an example, for such values of the Marangoni number, Yin et al. ${ }^{32}$ showed that after attaining the first plateau region visible in Fig. 3, the velocity would rise again and tend to a second plateau). In order to assess expressly the influence of the domain extension for such values of the Marangoni number, we performed an additional simulation using a (two times) larger domain length for the case for Ma $=200$ (Fig. 4). 


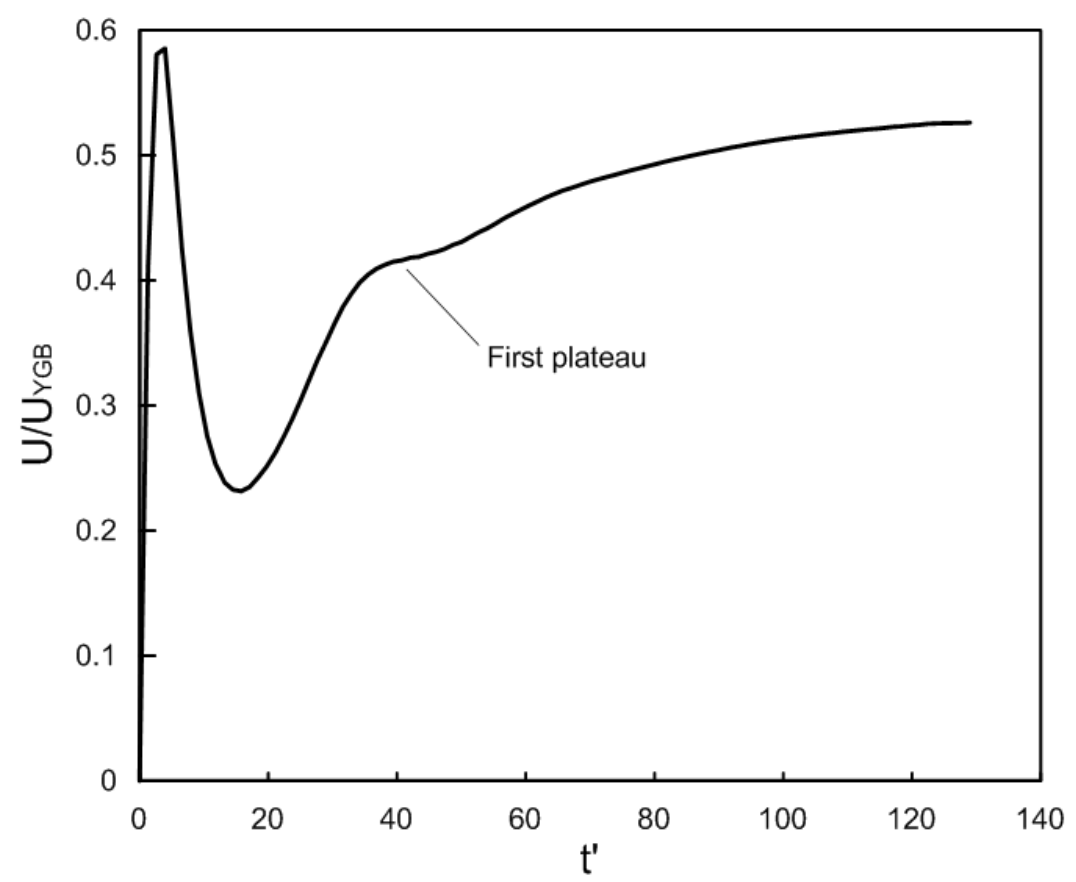

FIG. 4: Normalised droplet velocity for $\mathrm{Ma}=200$ as a function of the dimensionless time in a domain two times longer than the one used for the previous simulations.

The results, summarised in Fig. 4, confirm that after the first plateau the droplet undergoes a second stage of acceleration and eventually its speed converges to a final steady state value (the final velocity obtained in our simulation has been found to be in good agreement with the result obtained by Yin et $a l .{ }^{32}$ in similar conditions).

In order to compare our numerical results with the experiments of Hadland et al., ${ }^{12}$ we followed the same procedure used in the experiments, i.e. we considered for comparison the velocity attained by the droplet at $z \sim 4 \mathrm{~cm}$. Fig. 5 summarises our results on the effect of Marangoni number on the scaled migration and compares them to previous works in the literature, showing the good agreement between our results and the experiments ${ }^{12}$ ). Notice also the agreement between our data and the simulations by $\mathrm{Ma}^{65}$ for Marangoni up to 100 . Above this value the two trends deviate considerably. Such differences might be explained by the limited extension of the geometry. As previously pointed out, for Marangoni numbers larger than 100 the distance covered by the droplet required to reach a final steady state increases considerably and, therefore during the experiments the droplet did not have enough time to reach the terminal velocity. By considering exactly the same domain of the experiments in our simulations, our results are able to capture correctly the experimental trend. 


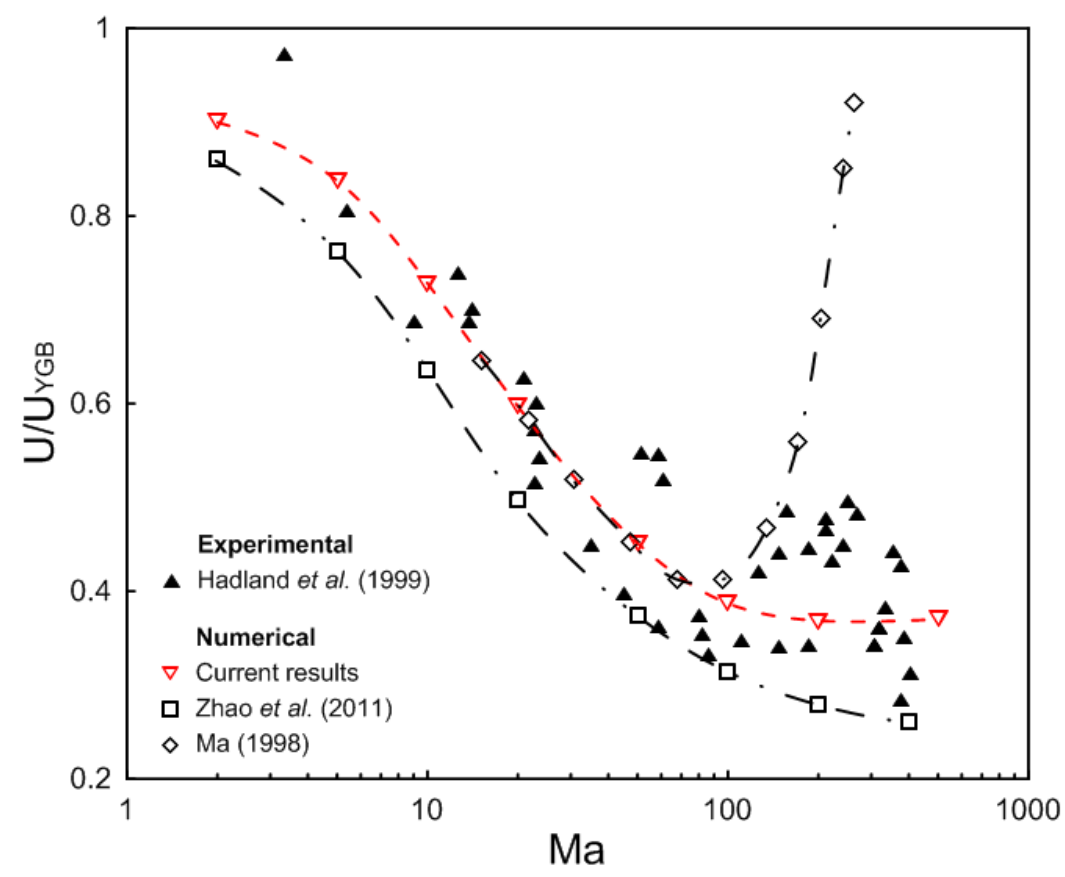

FIG. 5: Effect of the Marangoni number on the normalised velocity. The dashed red line represents a spline fitting to our numerical results (open triangles), the triangles represent experimental results; and the open symbols (squares and diamonds) correspond to numerical predictions from other authors.

\section{Wall effects}

In this section, we study the dynamics of the wall-droplet interaction for the same parallelepipedic domain, same droplet radius and fluid properties adopted in the previous section corresponding to the experimental set-up of Hadland et al. ${ }^{12}$ However, to assess the effect of the proximity to the wall, we performed a series of numerical experiments releasing the drop at the same location along $z$, but with an initial "off-set" position with respect to the centre of the channel as schematically shown in Fig. 6. In this section, we analyse the case of an off-centred droplet by varying its initial position in the $x$-direction (this situation is referred to as the droplet-near-side "NS"case, Fig. 6a) and in the following section (Sect. III A3), we look at the "joint" effects produced by the proximity of the droplet to two sidewalls (by setting the droplet off-set in both $x$ - and $y$-directions, this configuration will be referred to as the droplet-near-corner "NC" case). To the best of our knowledge, no simulations or experiments have been expressly devoted to addressing this problem. Again, fully three-dimensional simulations have been performed. 


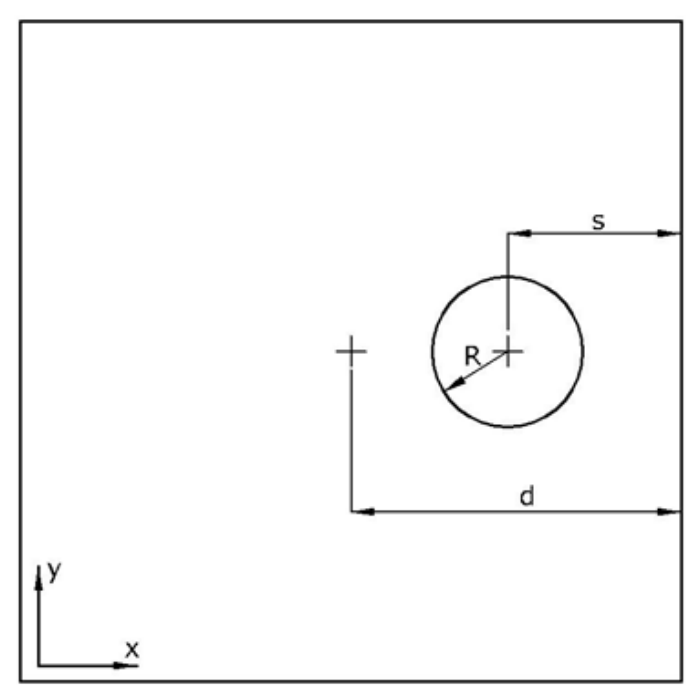

a)

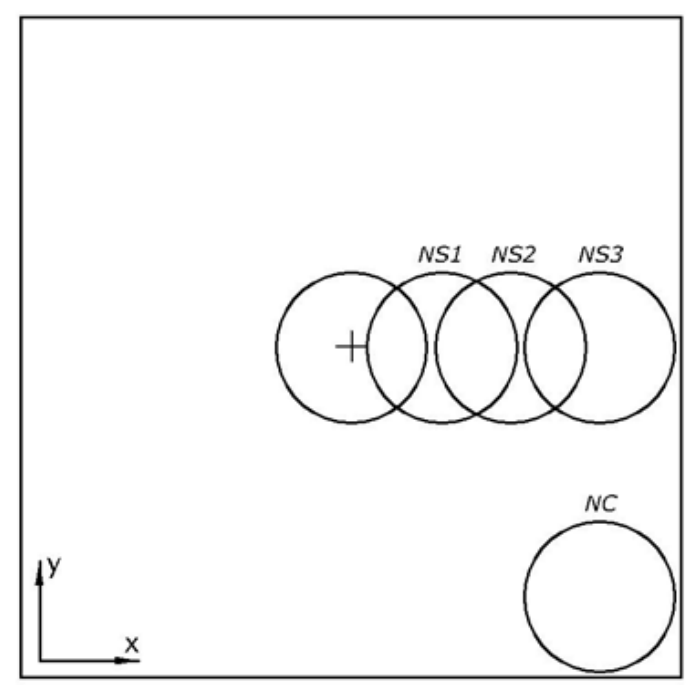

b)

FIG. 6: (a) Sketch of the domain in a plane normal to the direction of the motion: a) off-set droplet in its initial position, with relevant variables used to define its position. b) Initial position of the droplets for all the configurations considered.

In order to quantify the "proximity" of the droplet to the wall (i.e. the drop interface-wall distance), and to give a measure of how far its centre is set away from centre of the channel, conveniently, we define the following two dimensionless parameters: the "degree of proximity" $\theta=R /(s-R)$, and the "off-set parameter" $e=(d-s) / d$. These two quantities are obviously related to each other:

$\theta=\frac{1}{d / R(1-e)-1}$

In our analysis, we studied three different initial configurations $(e=0.25,0.50,0.73$, with the latter corresponding to a distance $d-s \sim$ ) in addition to the original case for $e=0$ (considered previously in Sect. III A1). In order to assess wall effects in two different cases for which the role of the temperature field is expected to be different, two distinct flow regimes with $\mathrm{Ma}=O(1)$ and $\mathrm{Ma}=O\left(10^{2}\right)$ are considered, namely $\mathrm{Ma}=2$ and $\mathrm{Ma}=100$, (notice that for the matrix liquid $\operatorname{Pr} \approx 100$, therefore the Reynolds number is $<O(1)$ and $=O(1)$, respectively). While in the first case, both the convective transport of momentum and energy can be assumed to be negligible (creeping flow conditions), in the latter case they are not (especially the convective transport of heat, which is expected to produce significant distortions in the temperature field with respect to purely diffusive, i.e. thermally stratified, conditions).

The differences between these two regimes in terms of behaviour taken by the non-centred droplet can be clearly appreciated in Fig. 7, which shows the droplet migration velocity normalised using the velocity it would reach if it were released from the centre of the domain (i.e. the steady-state velocity for $e=0$ ) as function of the parameter $\theta$. 


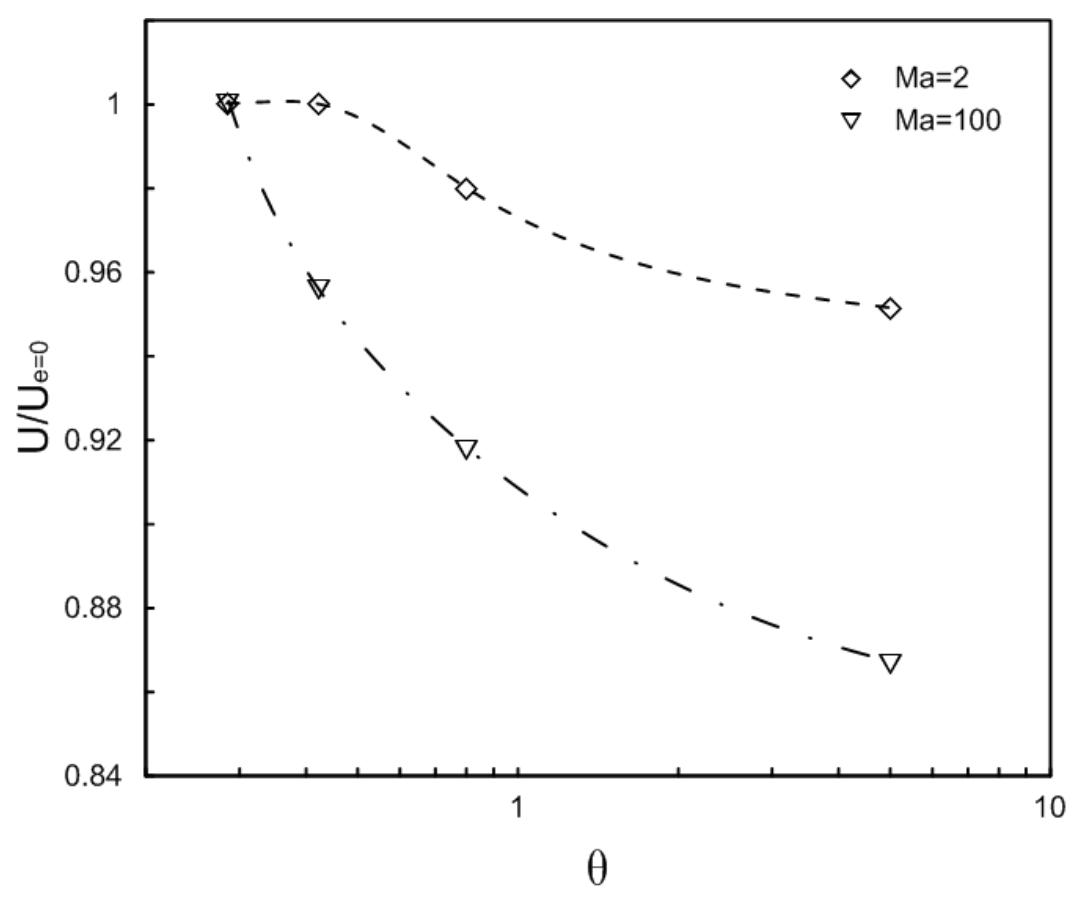

FIG. 7: Effect of $\theta$ on the normalised migration velocity for two different flow regimes. The lines are a guide to the eye.

The results indicate that in both regimes, as the drop is released in a position increasingly closer to the wall, the migration velocity decreases. However, such a decrease is enhanced for larger values of the Marangoni number. For $\mathrm{Ma}=2$ when $e=0.25(\theta=0.42)$, the droplet does not "feel" any wall effect. On the other hand, at $\mathrm{Ma}=100$, the droplet undergoes a significant decrease in speed even for relatively small values of $e$. Such results suggest that for the particular geometry adopted in the experiments of Hadland et al., ${ }^{12}$ for large Ma, even relatively small departures from the condition of perfectly centred droplet might influence the observed droplet dynamics and velocity of migration. According to our data, the following complex scaling law can be crafted for $\theta \leq 2$ : velocity scaling as $c_{1}+c_{2} \exp \left(\theta^{\mathrm{n}}\right)$, where $c_{1}$ and $c_{2}$ are two constants depending on the value of the Marangoni number and the exponent $\mathrm{n}$ takes values 1 and 2 in the creeping flow and $\mathrm{Re}=\mathrm{O}(1)$ regimes, respectively. 

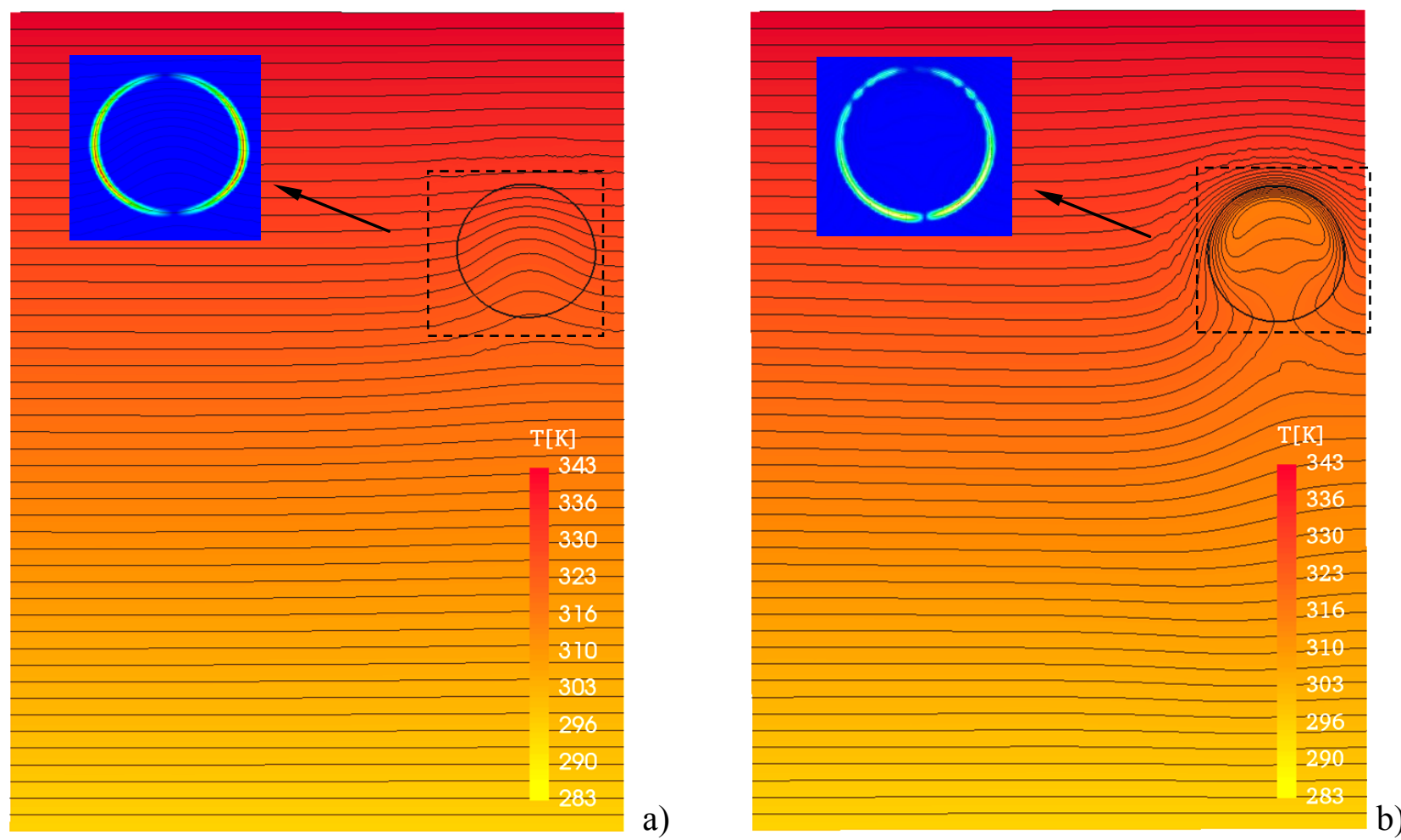

FIG. 8: Temperature field and thermocapillary force $\left(\mathrm{N} \times \mathrm{m}^{-3}\right)$ contours in the case $e=0.73$ for a) $\mathrm{Ma}=2, \mathrm{~b}$ ) $\mathrm{Ma}=100$. For $\mathrm{Ma}=2$, the thermocapillary force varies from a minimum of 0 (blue contour) to a maximum of $0.177 \mathrm{~N} \times \mathrm{m}^{-3}$ in the region of the interface. For $\mathrm{Ma}=100$, the thermocapillary forces varies from 0 to about $6.4 \mathrm{~N} \times \mathrm{m}^{-3}$ in the region of the interface.

As anticipated, this scenario can be explained by considering the specific behaviour of the temperature field. At $\mathrm{Ma}=2$, the temperature field attains a quasi-linear distribution (see Fig. 8a for the case in which the droplet was released closest to the wall, $(e=0.73)$. In such circumstances it is clear that most of the deceleration produced by the proximity to the side wall has to be ascribed to kinematic effects (the increased viscous drag to which the droplet is subjected owing to its interaction with the side wall, which leads to an increase in the opposing shear stresses in the fluid region between the droplet and the wall). For larger values of the Marangoni number, however, the distortion of the temperature field due to its interaction with the droplet motion becomes significant and this, in turn, has a back effect on the velocity of the droplet itself (when the droplet is located close to the wall, such a proximity has an impact on the thermal field, which becomes highly distorted as shown in Fig. 8b). As a natural consequence, the distribution of thermocapillary stresses at the droplet surface changes with respect to the case in which the droplet is far from the wall (most remarkably, the resulting Marangoni force is no longer oriented along a direction parallel to the imposed temperature gradient leading to the emergence of a droplet velocity component perpendicular to the wall boundary as shown in Figs. 9 and 10). Hence, as the Marangoni number increases there are two different factors contributing to the decrease in velocity experienced by the droplet, one of purely viscous nature and another of a thermal origin. 


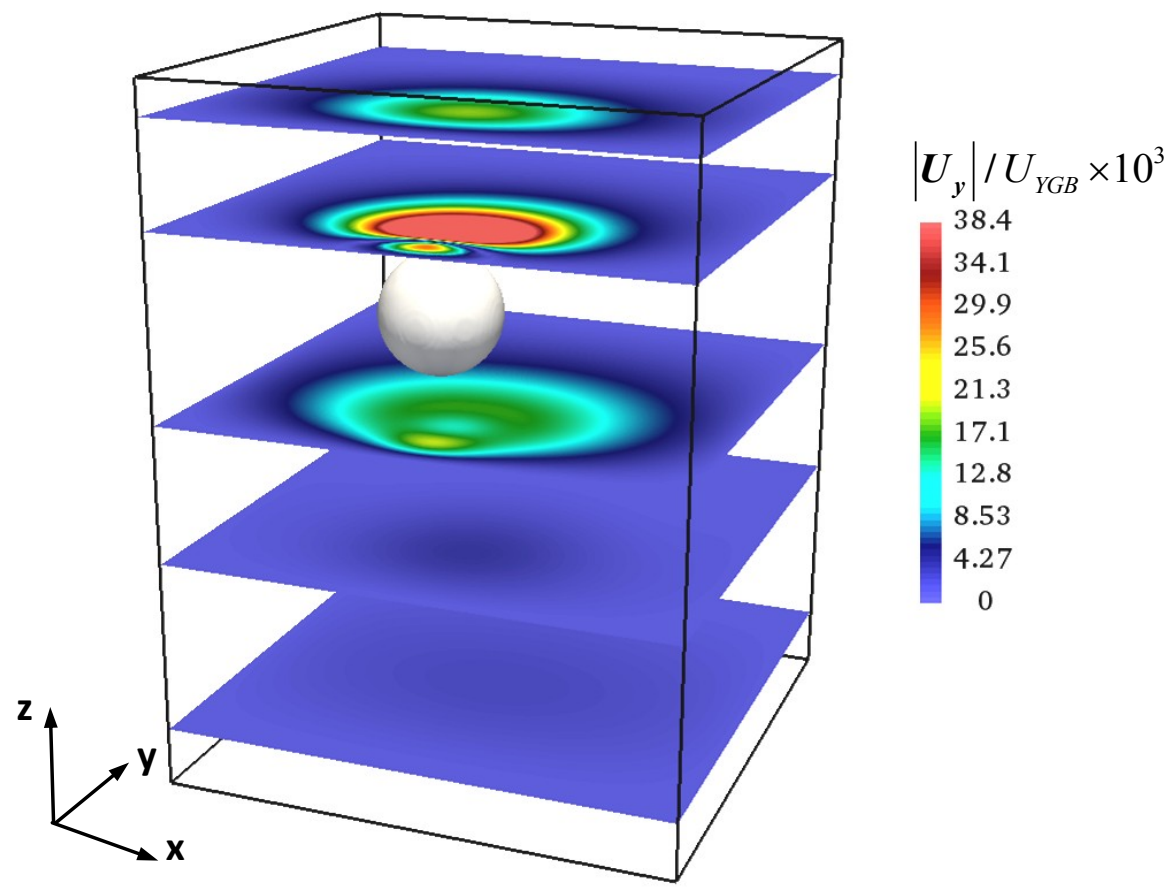

FIG. 9: $3 \mathrm{D}$ view of the scaled $y$-velocity component for $\mathrm{Ma}=100$ for five different $x y$-planes taken at $\sim 1.2 \mathrm{~cm}$ of distance between each other $\left(N S_{3}\right)$.

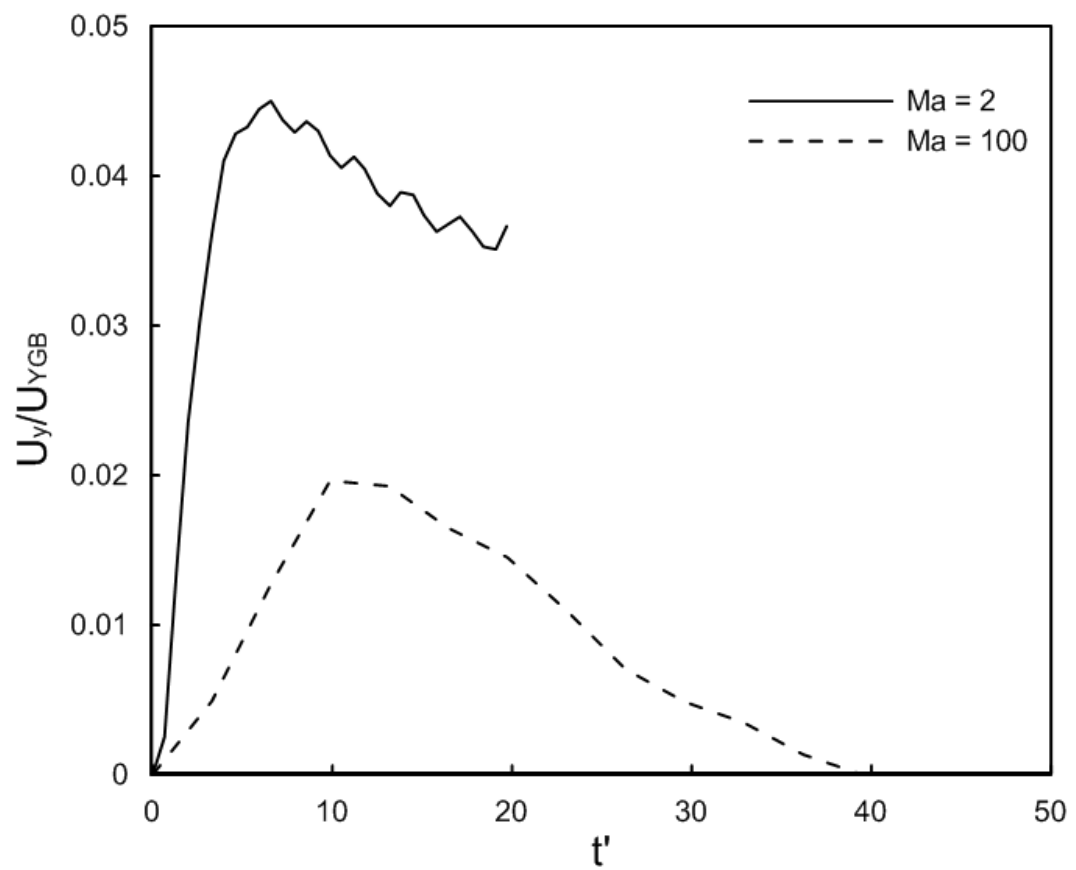

FIG. 10: Time evolution of the droplet $y$-velocity component normalised with the Young limit for the "NS 3 " cases at $\mathrm{Ma}=2$ and $\mathrm{Ma}=100$. Note that the numerical value is larger in the case of $\mathrm{Ma}=100$ compared for that at $\mathrm{Ma}=2$. The counterintuitive trend is due to the different value of the theoretical limit velocity used to normalise the effective droplet velocity in the two cases under discussion.

Interestingly, Fig. 10 shows an apparently oscillatory behaviour, which may indicate the onset of an instability (expected to be driven by the interplay between kinematic and shape deformation 
effects). This might be the subject of a future work entirely devoted to addressing such aspects, which are beyond the scope of the present study.

\section{Corner effects}

Since in a box-shaped three-dimensional domain, in principle, a drop may transit in a region located near the intersection of two adjacent walls (i.e. close to a corner), in the remainder of this section we expressly concentrate on such a case. In order to do so, we release the drop from an initial position constrained by two adjacent walls (indicated as case " $N C$ " in Fig. 6b); because the $z$-axis is the axis of droplet motion, in practice, this is equivalent to considering an equal "off-set" parameter in both $x$ - and $y$-directions. The migration velocity for the " $N C$ " configuration is compared with previous cases (Sect. III A1) with $e=0$ and $e=0.73$ and shown in Figs. 11 and 12, for a range of Marangoni numbers. Fig. 11 provides the scaled droplet speed as function of the dimensionless time for three different configurations and several values of the Marangoni number.
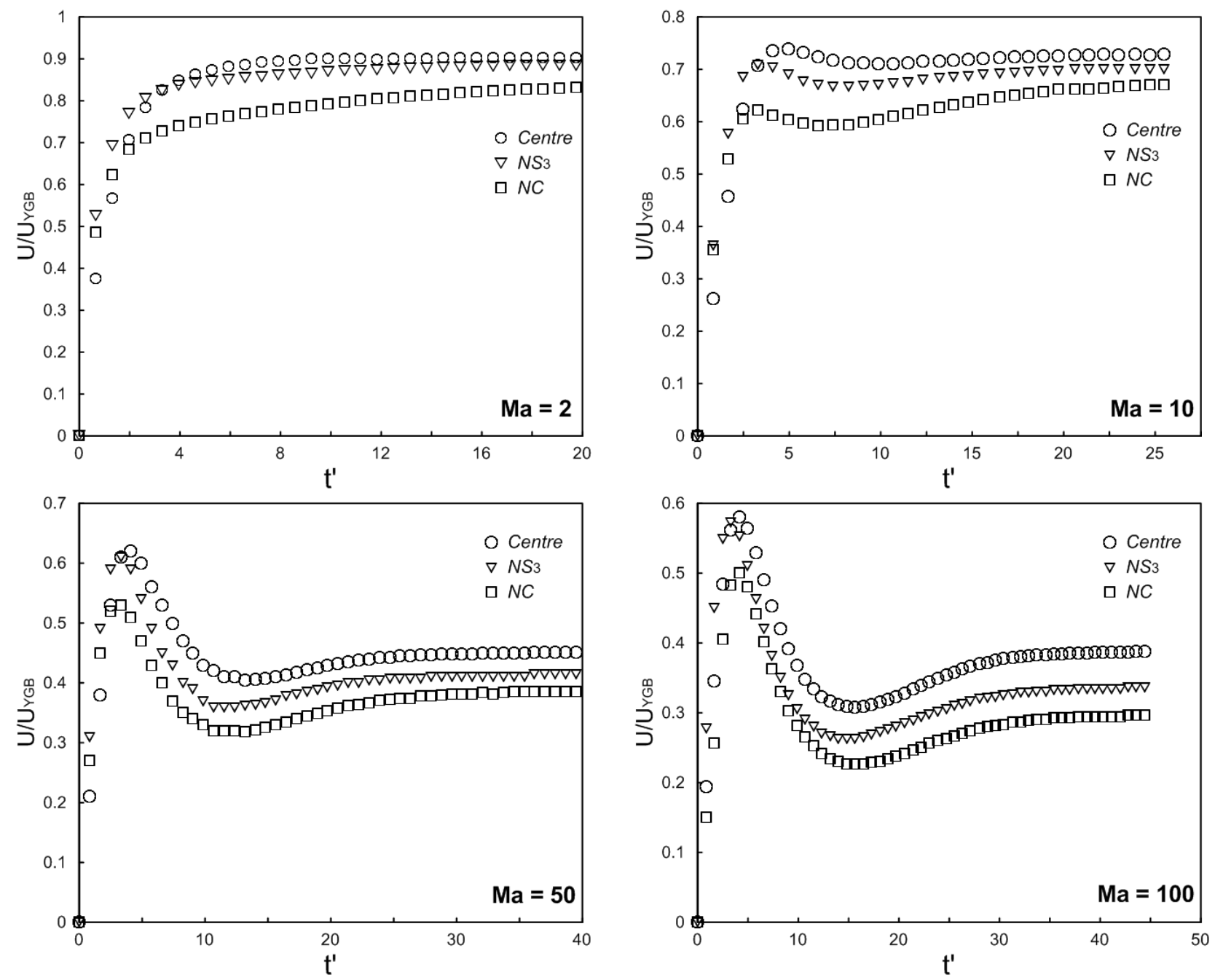
FIG. 11: Time evolution of the droplet velocity normalised with the Young limit for the three cases considered: $e=0$ ("centre"), $e=0.73$ along the y-axis ("NS 3 ") and $e=0.73$ along both $x$ and $y$ axis $(N C)$.
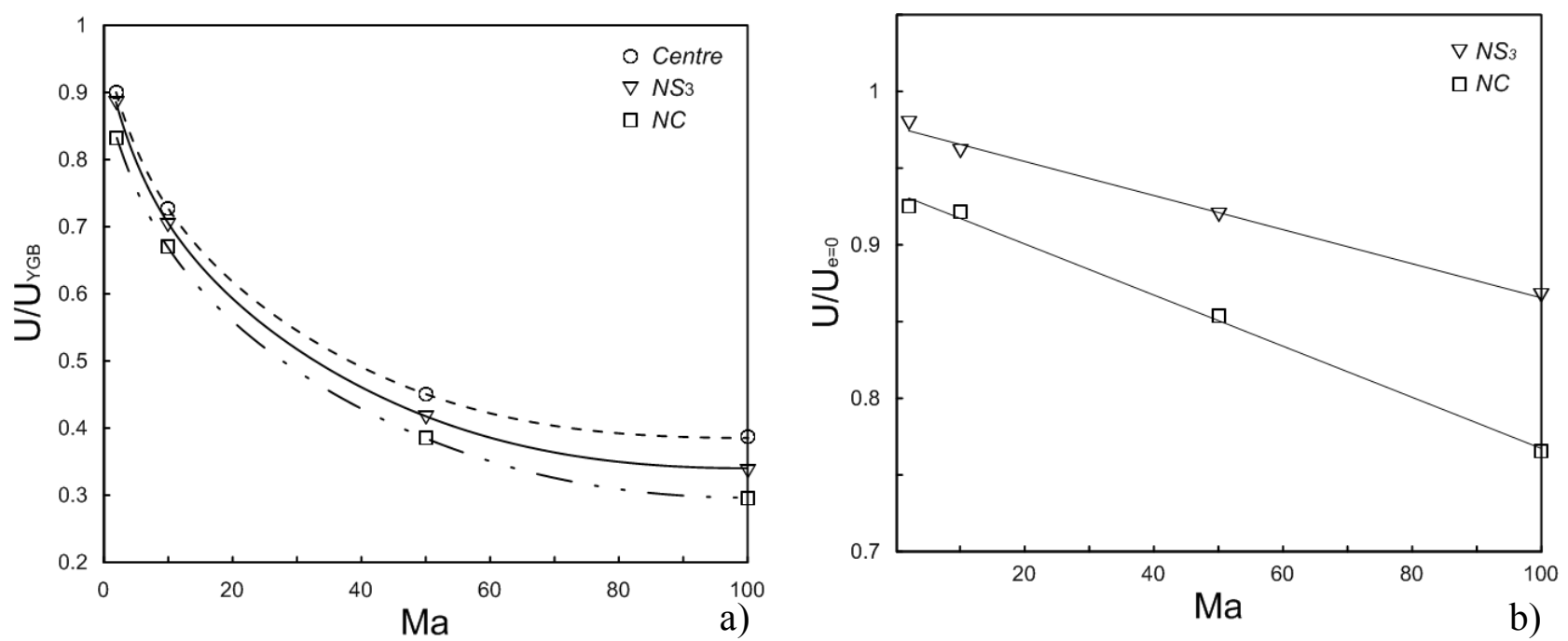

FIG. 12: a) Comparison between the scaled migration velocity for $e=0$ (centre) and $e=0.73\left(N S_{3}\right.$ and $N C$ ). b) Scaled velocity normalised with its counterpart for $e=0$, for different Marangoni numbers $(\mathrm{Ma}=2,10,50,100)$.

The most interesting information in these figures is the evidence they give about the enhanced droplet "slowing down" effect when the droplet can "feel" the presence of two distinct (adjacent) walls. Such behaviour can be justified considering that, as explained in Sect. III A2, due to the wallinduced distortion of the temperature field, the droplet experiences a lift force, which "pulls" it away from the boundary with a certain velocity. In this case, part of the available "driving force" is used to displace the droplet from the wall (instead of accelerating it in the direction of the imposed temperature difference). This interpretation is further confirmed in Fig. 12 where the asymptotic migration velocity of the droplet-near-corner case $(e=0.73)$, normalised by its counterpart at $e=0$ has been reported as a function of the Marangoni number. It can be noticed that the distance between the two lines attains a minimum for $\mathrm{Ma}=2$ (where the decrease in the velocity can be ascribed only to viscous effects) and increases with the Marangoni number due to the aforementioned thermal effect. A $3 \mathrm{D}$ view of the scaled velocity magnitude $\sqrt{U_{x}^{2}+U_{y}^{2}} / U_{Y G B}$ (considering the velocity components in a plane perpendicular to the temperature gradient) for $\mathrm{Ma}=100$ is shown in Fig. 13. 


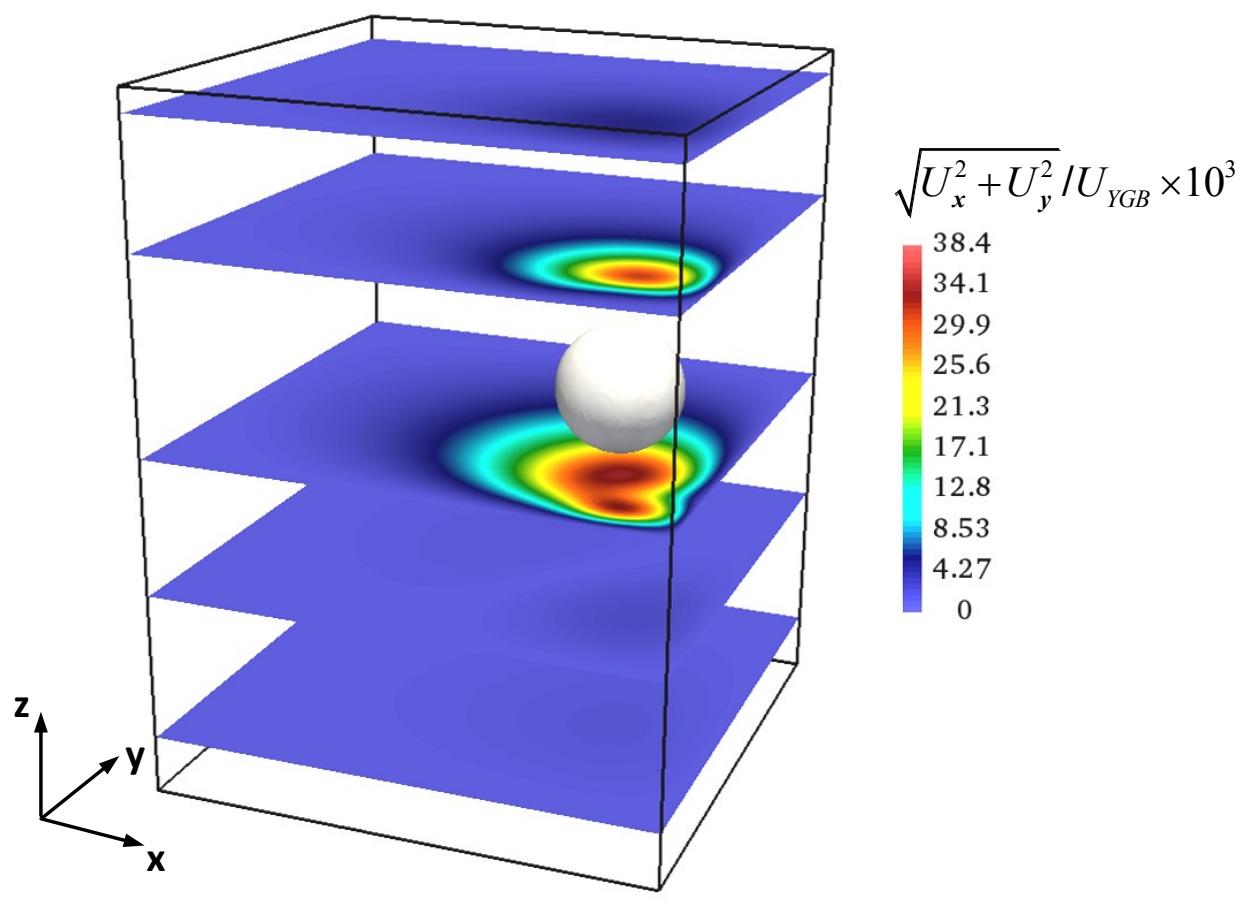

FIG. 13: 3D view of $\sqrt{U_{x}^{2}+U_{y}^{2}} / U_{Y G B}$ for 5 different $x y$-planes taken at $\sim 1.2 \mathrm{~cm}$ intervals for $\mathrm{Ma}=100(N C)$.

\section{Wall effects in non-adiabatic conditions}

In Sect. 3.A. 2 we considered the migration of the droplet near a wall assuming perfectly adiabatic conditions. In order to assess the role potentially played by the thermal boundary condition assumed at the walls, additional simulations (for some representative cases) have been performed replacing the adiabatic conditions with an alternate condition valid in the opposite limit in which the boundaries behave as purely conducting walls (while from an experimental standpoint, adiabatic conditions would be maintained only by an exceptionally insulating material, conducting conditions can be easily implemented in experiments by using walls made of a metal). Towards this end, we have imposed a constant linear temperature distribution on the boundaries (in practice, the same linear profile established at the beginning of the calculation) and performed new simulations for the case at $\mathrm{Ma}=2$ and $\mathrm{Ma}=100$ (along these lines, Figures 14a and 14b show the normalised droplet velocity and normalised distance from the wall relative to that evaluated at the initial time for both the "adiabatic" and "conducting" cases). As evident in these figures, when the convective transport of energy is negligible ( $\mathrm{Ma}=2$ ), the difference between the two cases is limited to a quantitative modification of the droplet velocity. In each situation the droplet moves further away from the wall with a velocity that is roughly constant, thus the droplet describes a linear trajectory. However, the velocity component that "pulls" the droplet away from the wall is smaller than that for the adiabatic-wall case (as witnessed by the different terminal velocity and the different inclination of the related trajectory in Figure 14a). 

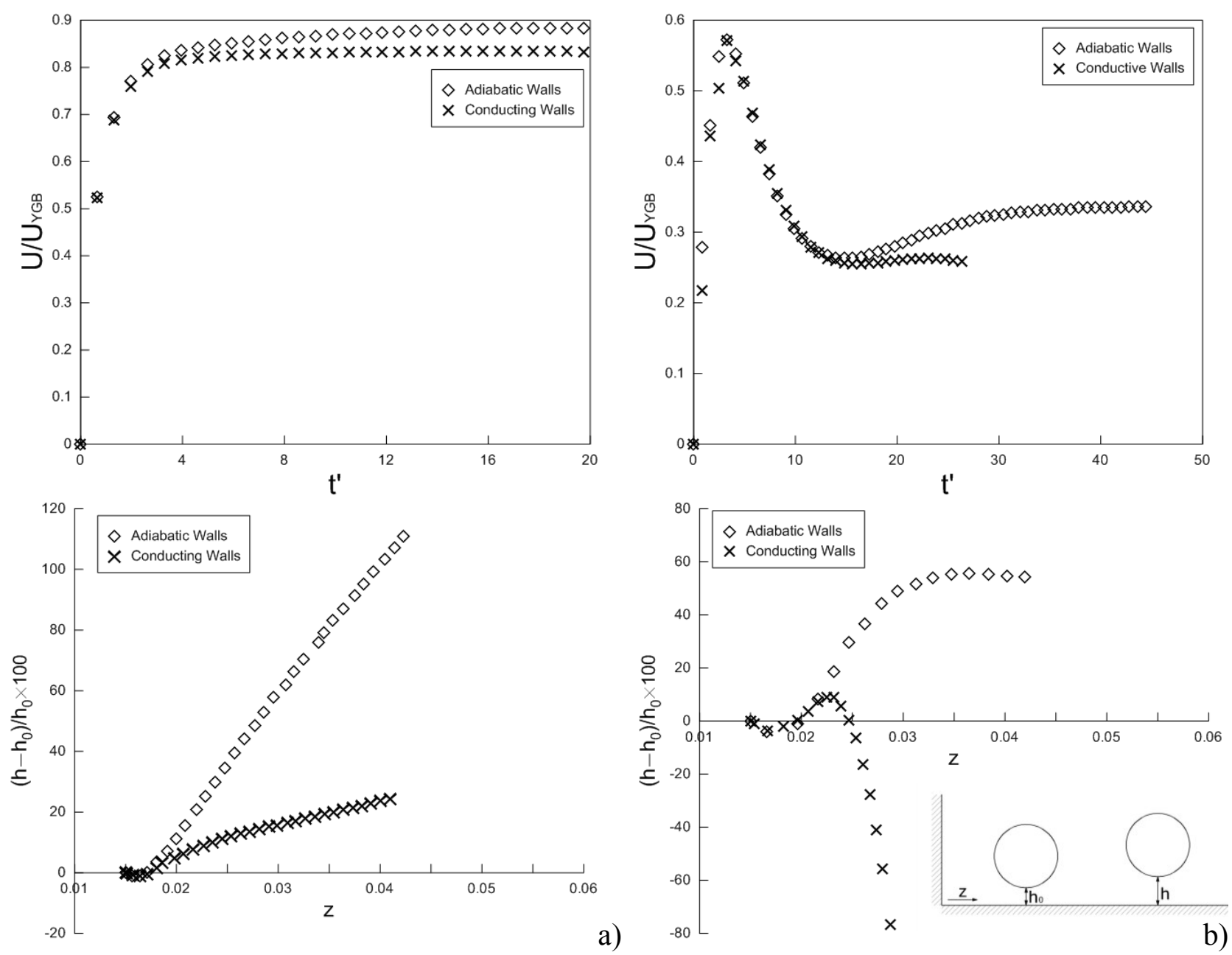

FIG 14: Normalised droplet velocity (top) and distance (bottom) from the wall relative to that evaluated at the initial time for both the "adiabatic" and "conductive" cases at $\mathrm{Ma}=2$ (a) and $\mathrm{Ma}=$ 100 (b).

The differences become much more striking for $\mathrm{Ma}=100$, with and the dynamics exhibiting a very different trend according to the thermal boundary condition considered. For the adiabatic wall, the droplet initially follows a linear trajectory that is qualitatively similar to the one observed for the case $\mathrm{Ma}=2$, then the normal velocity component vanishes and the trajectory becomes roughly parallel to the wall. By contrast, in the conducting-wall case, the droplet initially goes away from the side, but at a certain stage the velocity component perpendicular to the boundary reverses its sign. When this happens, the droplet starts to move very quickly towards the wall until it touches it. These behaviours are driven by the prevailing temperature gradient across the droplet and related distribution of thermocapillary stresses.

This gradient has two distinct components, namely an axial component (causing the droplet to migrate from the cold wall to the hot wall) and a "perpendicular" component induced by the droplet interaction with the wall and related thermal conditions. As revealed by the simulations, for $\operatorname{Re} \cong 0$, i.e. $\mathrm{Ma}=2$, a change in the nature of the thermal boundary conditions does not lead to qualitative modifications in the behaviour of the droplet (indeed, for such conditions the departure of the temperature field from linear diffusive conditions is almost negligible regardless of the thermal conditions at the wall). Nevertheless, for $\mathrm{Ma}=100$, the assumption of conducting wall causes a sign reversal in the component of the temperature gradient perpendicular to the solid boundary. 
Such behaviour can be clarified by observing the distribution of the isotherms (that in turn affect the thermocapillary stresses) shown in Fig. 15, top. When the wall is adiabatic, the isotherms are symmetrically distributed around the axis " $\zeta$ " of the drop and consequently the Marangoni stresses are also roughly symmetric and the resulting integral is a vector that points toward the direction of the axis of symmetry of the container (cf. the vector $\mathbf{F}_{\mathrm{MS}}$ at the bottom of the same figure). In the presence of a conductive wall, on the contrary, the thermocapillary stresses are no longer symmetrically distributed because the temperature field in the area near the wall is different from that established on the opposite side. In particular, we can imagine to subdivide the droplet in three different regions: an "upper" area, away from the wall (region I as shown in Fig.15), and two "bottom" areas near the wall (region II and III). We notice that in the upper part the temperature field is similar to that established in the case with adiabatic conditions. The lower parts, however, are considerably different. In region II the isotherms tend to "embrace" the surface of the droplet as a consequence of the temperature of the wall being imposed. Consequently, in that area the temperature gradient is mainly directed perpendicularly to the interface and has a strong component perpendicular to the solid wall as well. This gradient obviously gives only a little contribution in terms of thermocapillary stresses. On the contrary, in region III the isotherms are distributed roughly normally to the interface, therefore their contribution to the thermocapillary stresses is maximised (in this case the temperature gradient is mainly directed tangentially to the interface). This uneven stress distribution is responsible to the occurrence of a net force with a component directed toward the surface (see the vector $\mathbf{F}_{\mathrm{MS}}$ depicted in the bottom figure) that eventually causes the droplet to approach the boundary.

In the case of an actual experiment, where the side walls are expected to behave intermediately between the two ideal conditions considered here, one may expect the droplet to behave in a manner somehow intermediate, depending on the effective thermal flux established between the fluid and the external environment and the relative droplet-wall distance.
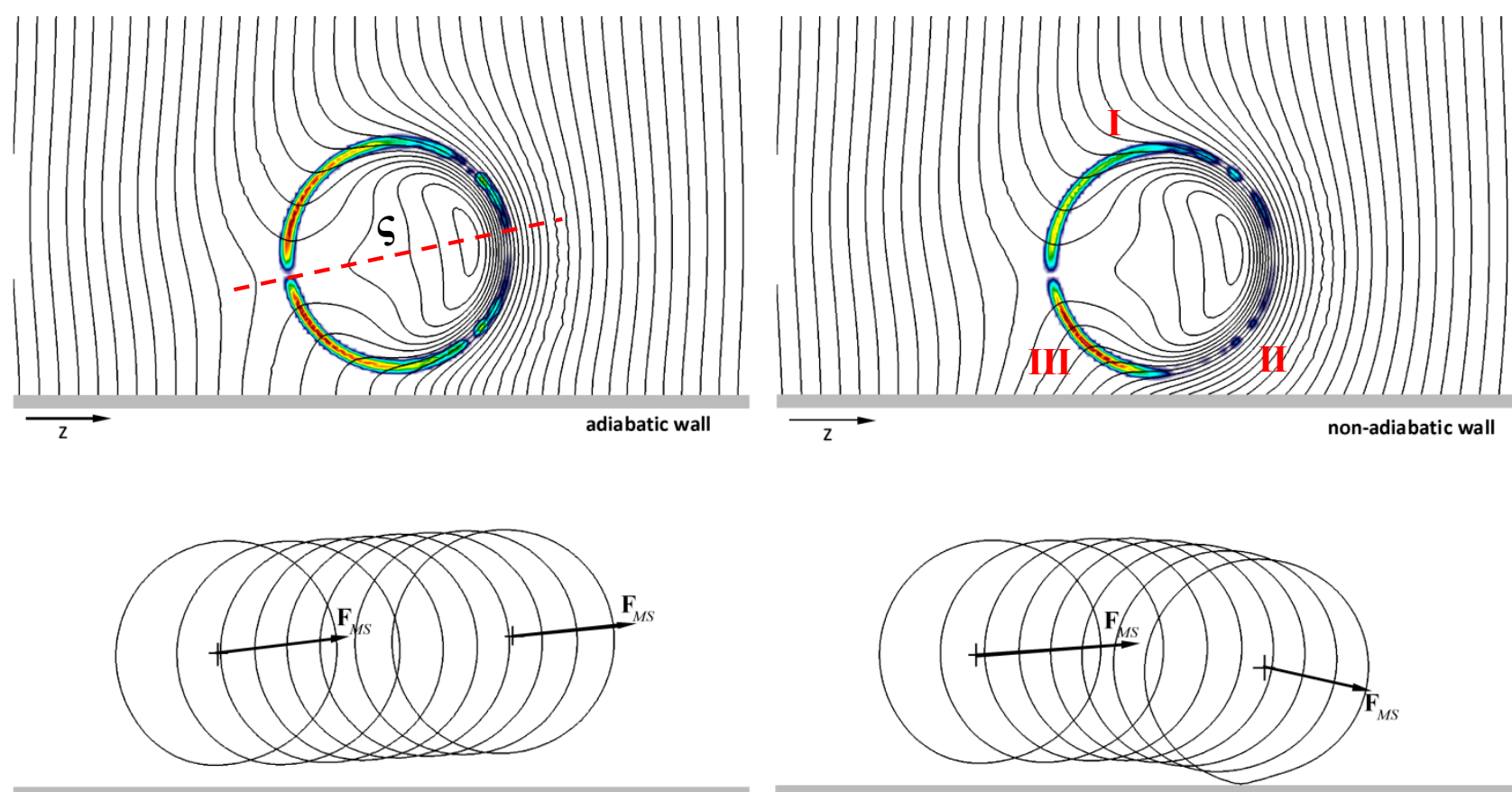
FIG 15: Comparison between the thermal field with superimposed thermocapillary stresses around the droplet (top) and trajectories (bottom) in the " $N S$ " case and for $\mathrm{Ma}=100$ in the case of adiabatic and non-adiabatic walls.

\section{B. Droplet migration in 3D converging and diverging geometries with adiabatic sidewalls}

In this section, we consider a set-up similar to the previous parallelepipedic container, but with the widths of the square cross-section decreasing (converging case) or increasing (diverging case) linearly along the domain from the cooled to the heated wall. Accordingly, we define the average container width $\bar{w}$ as $\bar{w}=\left(w_{\text {hot }}+w_{\text {cold }}\right) / 2$ where $w_{\text {hot }}$ and $w_{\text {cold }}$ are the dimensional widths of the hot and cold side, respectively. The overall system aspect ratio ( $A R$ ), in turn, is introduced as its length-to-average-width ratio ( $A R=$ ) (refer to Fig. 16). Another relevant characteristic geometrical parameter is the expansion ratio $E R=$ old, which can be greater than 1 (diverging geometry) or smaller than 1 (converging geometry), while for $E R=1$ one would recover the classical case with parallel boundaries considered in earlier sections.

Such cases are of special interest because it might be argued in advance that for such containers the temperature field has to play a significant role even under creeping flow conditions.

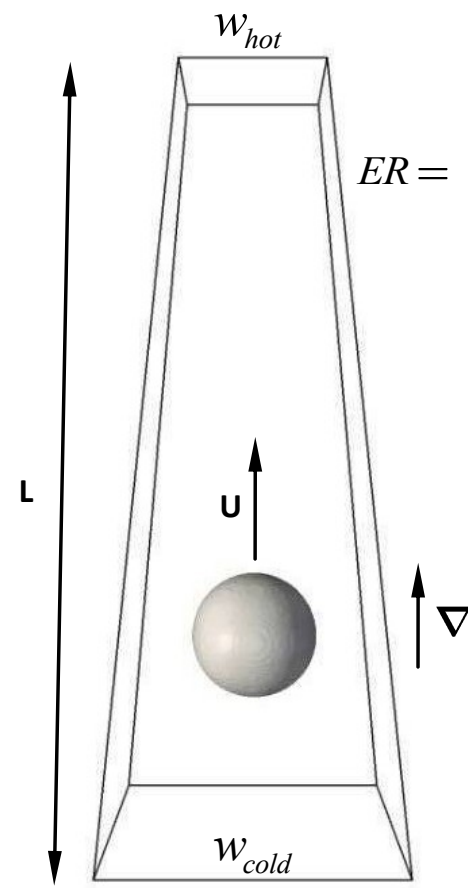

a)

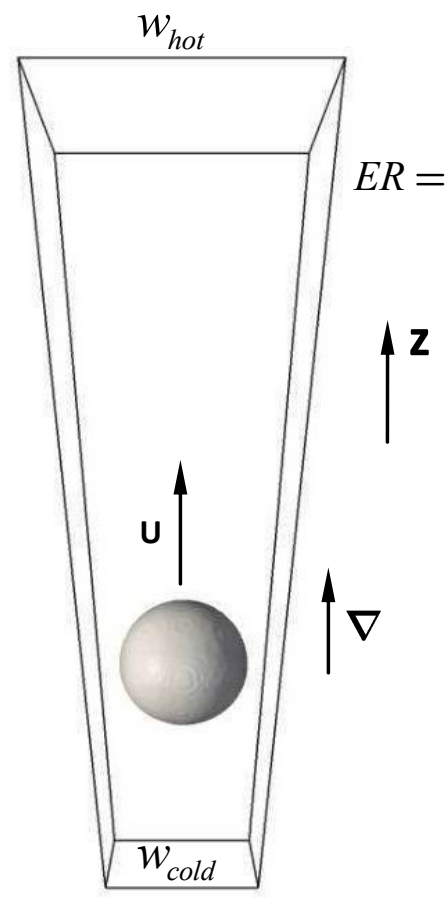

b)

FIG. 16: Schematics of the container with converging a) and diverging b) adiabatic sidewalls.

In such a context, before starting to deal with the droplet migration problem, it is instructive to consider the behaviour of the temperature field in the limit as $\mathrm{Re} \rightarrow 0$. For such a case, indeed, an analytic solution can be found for the temperature field. 
Our interest in such an expression stems from a two-fold purpose. First, it can be used to show that the assumption of linear temperature increase along the vertical ( $z$-axis) valid in the case of straight containers with uniform cross-section, no longer holds. Second, such an analytical expression can be used as a relevant initial condition to accelerate the convergence of the algorithm relating to the numerical solution of the momentum and mass conservation equations in the more general case for which both Re and Ma have finite values and $E R \neq 1$.

Most conveniently, we start from the balance of energy cast in its simplest form, that is, the conservation of energy flux along the horizontal direction:

$$
w^{*}(z) \frac{d T^{*}}{d z^{*}}=
$$

where $T^{*}$ in this context is the non-dimensional temperature defined as $\left(T-T_{\text {cold }}\right) /\left(T_{\text {hot }}-T_{\text {cold }}\right), T$ is the dimensional temperature and $w^{*} z^{*}$ is the nondimensional extension (using the average width $\bar{w}$ as reference length) of the generic cross section, which in turn can be expressed as:

$$
w^{*}\left(z^{*}\right)=w_{\text {cold }}^{*}+\left[\frac{w_{\text {hot }}^{*}-w_{\text {cold }}^{*}}{A R}\right] z^{*}=w_{\text {cold }}^{*}\left[1+(E R-1) \frac{z^{*}}{A R}\right]
$$

Substituting Eq. (19) into Eq. (18) gives:

$$
\frac{d T^{*}}{d z^{*}}=\frac{c_{1}}{w_{\text {cold }}^{*}\left[1+(E R-1) z^{*} / A R\right]}
$$

which, once integrated, yields:

$T^{*}\left(z^{*}\right)=\frac{c_{1} A R}{w_{\text {cold }}^{*}(E R-1)} \ln \left[1+(E R-1) z^{*} / A R\right]+c_{2}$

The two constants $c_{1}$ and $c_{2}$ can be determined forcing Eq. (21) to satisfy the boundary conditions at the two sidewalls, namely:

$z^{*}=0 \quad T^{*}=0 \rightarrow c_{2}=0$

$$
z^{*}=A R \quad T^{*}=1 \rightarrow c_{1}=\frac{w_{\text {cold }}^{*}(E R-1)}{A R[\ln (E R)]}
$$

Accordingly, Eq.(21) can be cast in compact form as:

$$
T^{*}\left(z^{*}\right)=\frac{\ln \left[1+(E R-1) z^{*} / A R\right]}{\ln (E R)}
$$


where, obviously:

$$
\lim _{E R \rightarrow 1} \frac{\ln \left[1+(E R-1) z^{*} / A R\right]}{\ln (E R)}=\frac{z^{*}}{A R}
$$

The temperature profiles obtained from eq. (23) (see Fig. 17) for different values of the parameter $E R$ clearly show a departure from the purely linear distribution of the temperature profile theoretically established for $E R=1$. The resulting profile is concave or convex for $E R<1$ or $E R>1$ respectively.
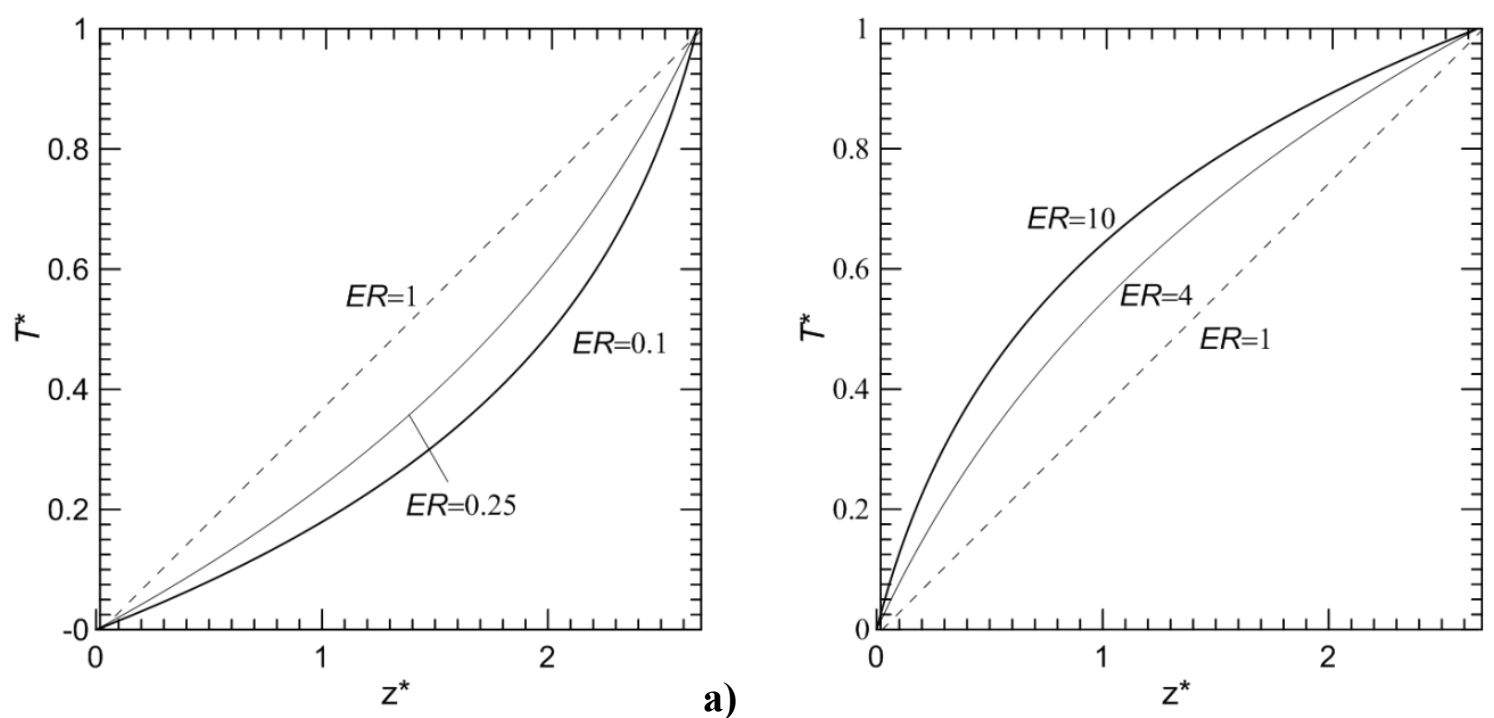

b)

FIG. 17: Exact solution for the temperature profile for $A R=2.66$ and $\mathrm{Re}=0$ (a dashed line is used for the corresponding ideal linear temperature profile obtained for $E R=1$ ): a) $E R<1$ (converging walls); b) $E R>1$ (diverging walls).

Fig. 18a shows the numerical results in the case of creeping flow (vanishingly small Marangoni and Reynolds numbers) for a converging and a diverging geometry with aspect ratio $A R=2.66$ together with the related results for the corresponding straight geometry ( $E R=1$ to be used for comparison). The latter is considered for three different values of the confinement parameter: $\theta=0.286$ (which corresponds to the geometry used in the experiments by Hadland et al. ${ }^{12}$ ), $\theta=0.42$ and $\theta=0.8$. For both converging and diverging geometries, the parameter $\theta$, evaluated in corresponding to the larger side is assumed to be $\theta=0.8$. 

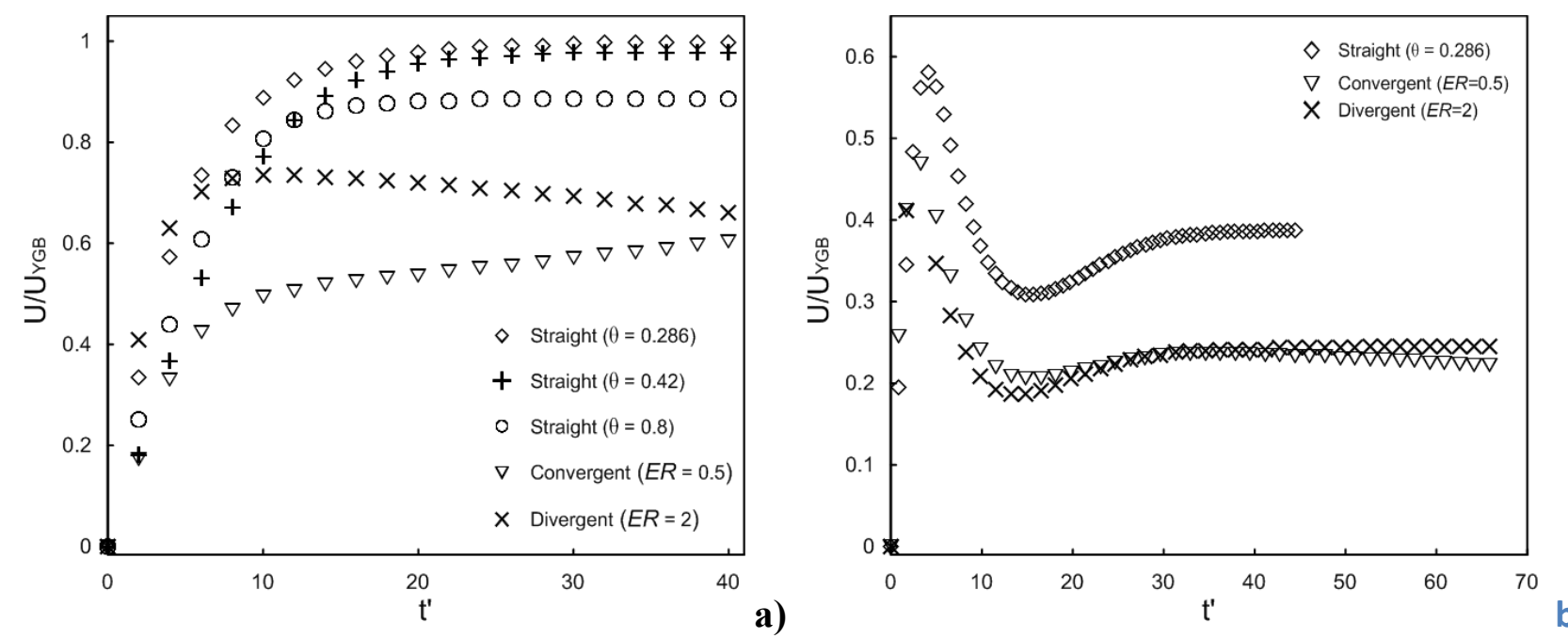

FIG. 18: Time evolution of the scaled droplet migration velocity for converging and diverging geometries (aspect ratio $A R=2.66$ ): a) in the limiting case of creeping flow compared to the droplet velocity for a "straight" geometry and different confinements; b) for $\mathrm{Ma}=100$ compared to the "straight" geometry used in the experiments of Hadland et al. ${ }^{12}$

The interpretation of the results shown in Fig. 18 is not as straightforward as first expected. On the basis of purely kinematic considerations, in the limit as $\operatorname{Re} \rightarrow 0$, one would expect the droplet to undergo deceleration in the converging channel case and vice versa for the diverging channel due to the frictional increased or reduced influence of walls, respectively. Nevertheless, Figure 18a shows just the opposite trend: droplet velocity increasing for a contracting geometry and decreasing when there is an expansion of the cross section (later, the droplets converge to a similar speed).

Such a counterintuitive scenario, however, can be justified on the basis of the arguments provided earlier about the behaviour of the temperature field in such geometries in the case $\operatorname{Re}<O(1)$. In such circumstances, the initial diffusive temperature profile is not significantly modified or disturbed by the migration of the droplet. As the temperature profile is convex for $E R>1$, the gradient is initially larger than that established in the case with $E R<1$ (see Fig. 17b) and this makes the droplet velocity (at least in an initial stage) higher than the corresponding migration velocity of the droplet in the geometry with converging walls (Fig. 18a).

As for this case the temperature gradient decreases as the droplet moves through the channel, its velocity progressively decreases accordingly. These trends are reversed for the case with converging walls (concave temperature profile, Fig. 17a). The temperature gradient is initially relatively small and then it increases as the droplet moves from the cold wall towards the hot one. This causes an acceleration of the droplet (Fig. 18a) (curve at $\mathrm{Ma}=0$ for the case $E R=0.5$ ). 


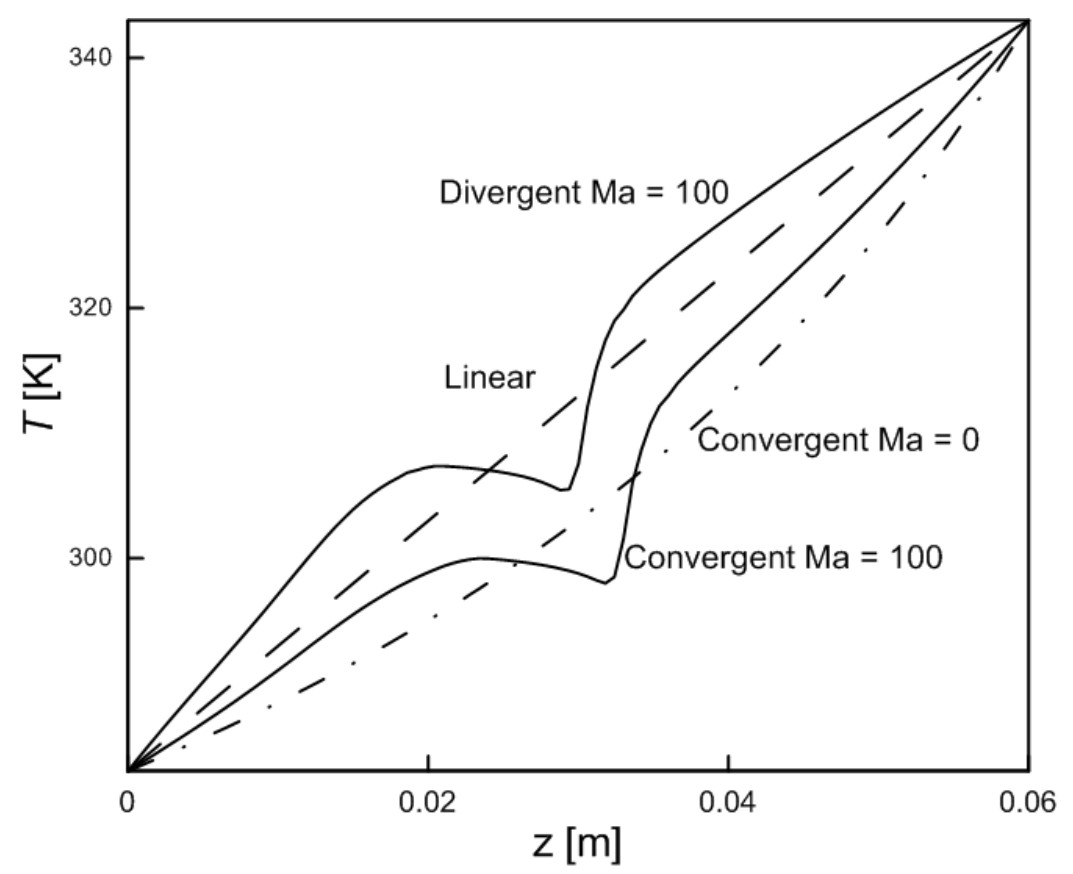

FIG. 19: Dimensional temperature profile along a line crossing the geometry in the middle of the channel for the converging $(E R=0.5)$ and diverging $(E R=2)$ geometries at $\mathrm{Ma}=100$ (solid lines), and temperature distribution for the convergent and box-shaped domains at $\mathrm{Ma}=\mathrm{Re}=0$ (dashed lines).

A considerable change however occurs when the convective transport of energy becomes more important. Figure $18 \mathrm{~b}$ shows the temporal evolution of the migration velocity for the two geometrical configurations under discussion when $\mathrm{Ma}=100$ (in the same plot we have also included the results about the straight channel for comparison). It can be seen that the two trends are qualitatively similar up to a dimensionless time t' $\sim 40$ (even though in the case $E R=2$ the velocity is always slightly higher). Most notably, after this instant the velocity of the droplet which is migrating within the converging channel starts to decrease at a constant rate (vice versa it was increasing in the case $\operatorname{Re}<O(1)$ ).

These behaviours can be explained by observing that, while for $\operatorname{Re}<O(1)$ the temperature gradients were the main "drivers" determining the droplet behaviours, we are now in the presence of several distinct effects. Along these lines, it is instructive to begin the related discussion by taking a look at Fig. 19, showing the temperature distribution on a segment passing through the centre of the domain for the two cases under discussion (in addition the "undisturbed" linear profile in the case of the straight channel and the profile for the converging channel when $\mathrm{Re} \rightarrow 0$ are also included). It can be seen that the temperature gradients in front of the drop for the case $E R=0.5$ are higher than those established in the case $E R=2$ like in the situation with $\operatorname{Re}<O(1)$; it can be noticed as well, however, that the temperature profiles are no longer logarithmic, and the differences in the gradients are much less pronounced than those seen for $\operatorname{Re}<O(1)$. This is also witnessed by the temperature fields shown in Fig. 20a (taken at the corresponding dimensionless time t' 40). Although the isotherms tend to be more condensed near the front of the drop in the case of 
converging geometry (which means that they give rise to larger Marangoni stresses), the related patterns for the converging and diverging geometries are qualitatively similar.

These mechanisms, however, are not the only ones at play in the considered dynamics.

For the case $E R=0.5$, as the droplet is forced to move through a converging geometry, at a certain stage it will feel an increasing effect of blockage due to the presence of the approaching walls. On the contrary, the scenario in the diverging channel is exactly the opposite: while the thermocapillary stresses are smaller because of the smaller temperature gradients, the droplet moves through a channel that offers a decreasing effect of blockage. In the light of these arguments, the similar trends of the migration velocity visible in the central part of the channel in Fig. 18b can be ascribed to these two effects compensating each other (in the second portion of the domain the two influential factors still "counteract": in the converging arrangement the propulsive temperature gradient and the blockage effect become increasingly larger while in the other situation the opposite applies).

To further clarify these aspects, Fig 20b shows the temperature gradient distribution. By inspecting this figure it becomes clear that near the interface (i.e. in corresponding to the vertical dashed lines) the temperature gradients are fairly similar because of the development of a thermal boundary layer in the front region and a thermal "wake" in the rear region; analogous considerations apply in the rest of the interface. It can be seen how the main thermal convection affects the temperature gradients at the droplet surface to make it less dependent on the value of the parameter $E R$ relative to the scenario seen for $\mathrm{Re} \rightarrow 0$. The differences between the temperature gradients for $E R<1$ and $E R>1$ are now less pronounced and, accordingly, the velocity evolutions are relatively similar in the central region of the channel. After a certain stage, however, the effect of blockage in the case $E R=$ 2 becomes predominant and the droplet inevitably starts to decrease.
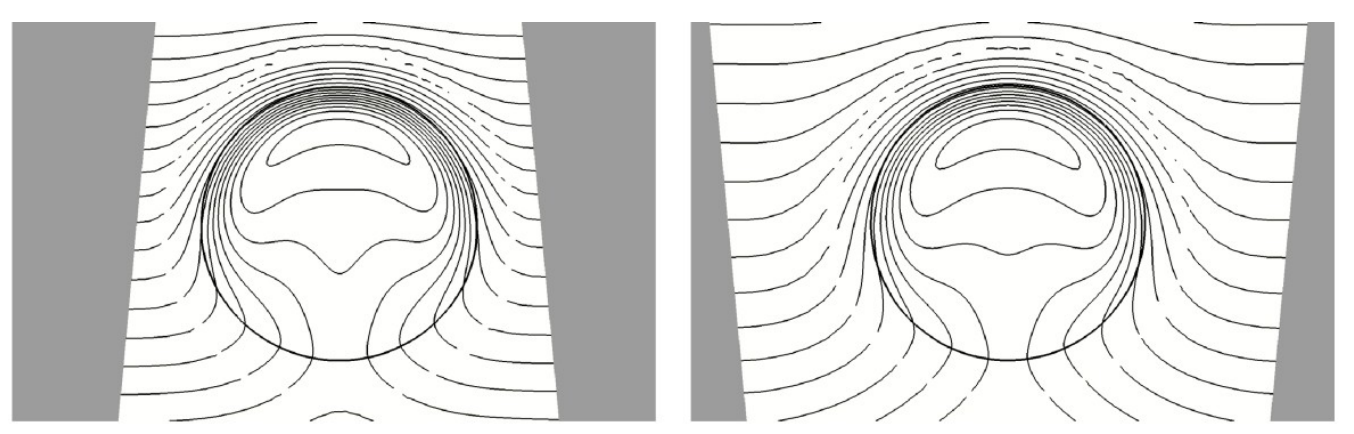

a) 


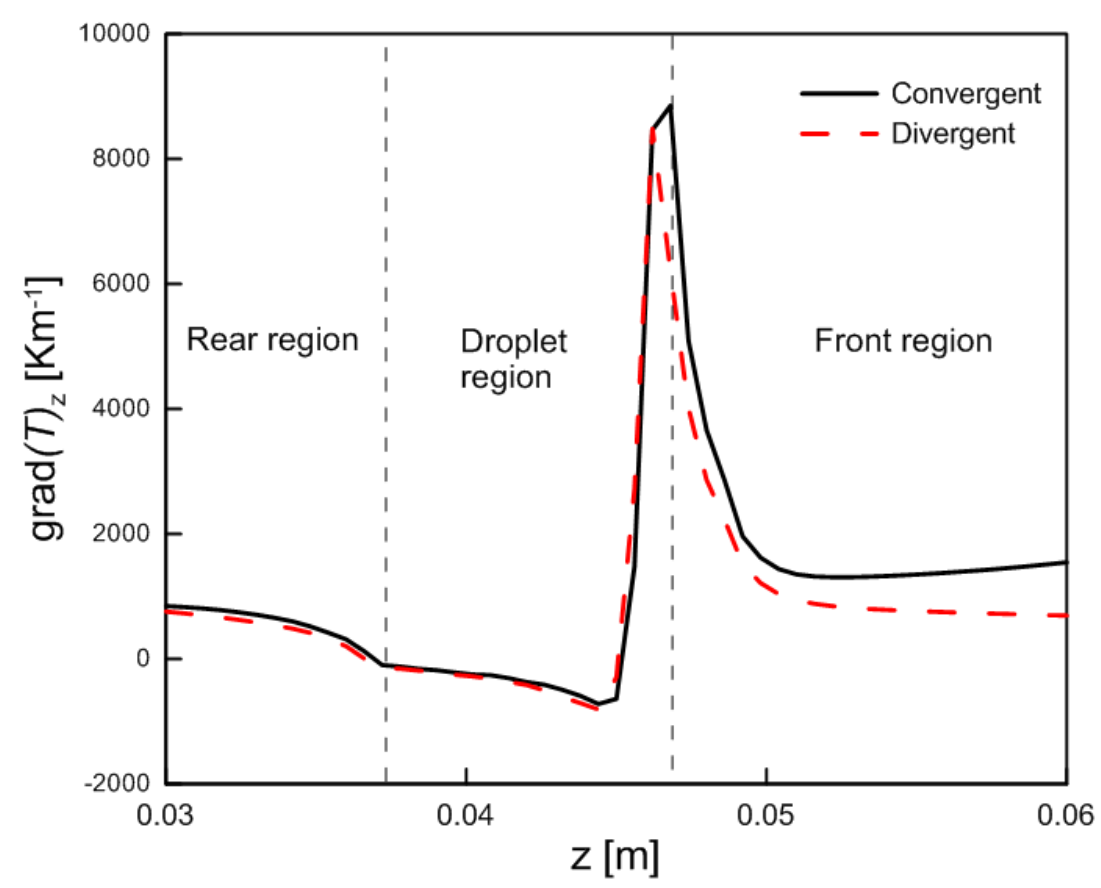

b)

FIG 20: a) Temperature distribution near the region of the droplet for the diverging (left) and converging (right) channels when $\mathrm{Ma}=100$ at $\left.\mathrm{t}^{\prime} \sim 65, \mathrm{~b}\right)$ Temperature gradient component evaluated along the line crossing the channel through its centreline.

On the basis of such results and related observations, therefore, we conclude that, while for $\mathrm{Ma}=O(1)(\operatorname{Re}<O(1))$ the difference between the straight geometry and the two cases with $E R \neq 1$ have to be ascribed essentially to thermal effects, in the case $\mathrm{Ma}=O(100)(\mathrm{Re}=O(1))$, the consequences of a non-linear temperature distribution are partially mitigated by the presence of thermal convection, which makes the temperature distribution around the droplet more or less the same regardless of the value of $E R$, thereby making blockage effects more influential in determining the velocity evolution.

\section{Conclusions}

The effect of the wall-droplet interaction and domain shape on the thermal Marangoni migration of droplets has been analysed in three-dimensional geometries using a coupled LS-VOF approach implemented in the framework of the OpenFOAM computational platform. We have studied several geometrical configurations, for different Marangoni numbers: droplet released in the proximity of a single adiabatic or purely conducting wall ( $N S$ " configuration) and droplet released in proximity of two adjacent adiabatic walls (i.e. close to a corner, " $N C$ " configuration). Moreover, the effect of the geometry shape has been accounted for considering the motion of the droplet in converging and diverging containers. All the computations have been performed allowing the Marangoni number to span a relatively wide range, with the extremes of such an interval corresponding to the situation of "creeping flow" (in which thermal effects are expected to play a negligible role) and a situation in 
which $\operatorname{Re} \geq O(1)$ (Ma up to 500 for which significant distortions in the temperature field are expected).

In the case of the wall-droplet interaction for adiabatic conditions, the results show that for both configurations ("NS" and " $N C$ ") the migration speed decreases with the Marangoni number when compared to the velocity one would observe for the droplet migrating in the centre of the channel. In addition, we have also noticed the presence of a velocity component directed along the direction perpendicular to the wall, which tends to "pull" the droplet away from the wall. We infer that such a velocity component is the result of wall-induced distortions present in the temperature field with increasing magnitude as the Marangoni number is increased (such distortions being weak or negligible when $\operatorname{Re}<O(1)$ where the only mechanism responsible for the droplet slowdown is of a viscous nature).

In the case of the interaction with a conducting wall ( $N S_{3}$ configuration), the results have revealed that the migration process is strongly sensitive to the value of the Marangoni number. More specifically, for $\mathrm{Ma}=2$ the scenario remains substantially unchanged with respect to the case with adiabatic walls, the main difference being related to a relatively small decrease in the velocity component that pulls the droplet away from the boundary. For $\mathrm{Ma}=100$ however, the distortion of the temperature field in the region between the droplet and the wall in the case of conducting sidewalls results in a uneven distribution of the thermocapillary stresses that is responsible for the occurrence of a net force with a component directed toward the surface (pulling the droplet towards the wall i.e. in the opposite direction with respect to that observed for adiabatic conditions).

With regard to the effect of the shape of the domain, our numerical experiments have revealed apparently counterintuitive results. For adiabatic sidewalls and $\operatorname{Re}<O(1)$ the temperature profile is essentially logarithmic (in line with the analytic solutions that can be obtained integrating the energy equation in the absence of convection) and the droplet undergoes acceleration or deceleration depending on the concavity of such a profile for $E R \neq 1$. When $\operatorname{Re}=O(1)$, however, the differences between the cases $E R<1$ and $E R>1$ in terms of temperature gradients are mitigated by the presence of strong thermal convection inside and around the droplet. Accordingly, the velocity displayed by the droplet in the two cases is relatively similar until blockage effects (due to the narrowing channel for the case $E R>1$ ) start to play a dominant role in the dynamics.

\section{Acknowledgments}

Part of the results were obtained using the EPSRC ARCHIE-WeSt High Performance Computer (www.archie-west.ac.uk). EPSRC grant no. EP/K000586/1.

\section{References}


[1] Lappa M. (2004), Fluids, Materials and Microgravity: Numerical Techniques and Insights into the Physics, 538 pages, Elsevier Science (2004, Oxford, England).

[2] Lappa M., (2005) Assessment of VOF Strategies for the analysis of Marangoni Migration, Collisional Coagulation of Droplets and Thermal wake effects in Metal Alloys under Microgravity conditions, Computers, Materials \& Continua, 2(1), 51-64.

[3] Arienti M., Sussman M., (2017), A numerical study of the thermal transient in high-pressure diesel injection, International Journal of Multiphase Flow, 88, 205-221.

[4] Esmaeeli A., Tryggvason G., (1998), Direct numerical simulation of bubbly flows. Part I - Low Reynolds number Arrays, Journal of Fluid Mechanics, 377: 313-345.

[5] Esmaeeli A., Tryggvason G., (1998), Direct numerical simulation of bubbly flows. Part II Moderate Reynolds number Arrays, Journal of Fluid Mechanics, 377: 313-345.

[6] Otha M, Sussman M., (2012), The buoyancy-driven motion of a single skirted bubble or drop rising through a viscous liquid, Physics of Fluids, 24(11), 112101.

[7] Direito F.J.N., Campos J.B.L.M, Miranda J.M., (2016), Rising of a single Taylor drop in a stagnant liquid-2D laminar flow and axisymmetry limits, Physics of Fluids, 28(5), 057101.

[8] Sousa R.G., PintoA.M.F.R., Campos J.B.L.M., (2007) Interaction between Taylor bubbles rising in stagnant non-Newtonian fluids, International Journal of Multiphase Flow, 33(9), 970-986.

[9] Behjatian A, Esmaeeli A., (2014), Confinement effects on electrohydrodynamics of twodimensional miscible liquid drops, Colloids and Surfaces A: Physicochemical and Engineering Aspects, 441, 116-126.

[10] Nguyen N., Ng K. M., Huang X., (2006), Manipulation of ferrofluid droplet using planar coils, Applied Physics Letters 89, 052509.

[11] Brunet P., Baudoin M., Bou Matar O., Zoueshtiagh F., (2010), Droplet displacement and oscillation induced by ultrasonic surface acoustic waves: A quantitative study, Physical Review E, $81,036315$.

[12] Hadland P.H., Balasubramaniam R., Wozniak G., Subramanian R.S., (1999), Thermocapillary migration of bubbles and drops at moderate to large Marangoni number and moderate Reynolds number in reduced gravity, Exp. Fluids, 26: 240-248.

[13] Esmaeeli A., (2005), Phase distribution of bubbly flows under terrestrial and microgravity conditions, Fluid Dynamics and Material Processing, 1(1): 63-80.

[14] Lappa M., Piccolo C., Carotenuto L., (2004), Mixed buoyant-Marangoni convection due to dissolution of a droplet in a liquid-liquid system with miscibility gap, European Journal of Mechanics/B Fluids, 23/5: 781-794.

[15] Lappa M. and Piccolo C., (2004), Higher modes of Mixed Buoyant-Marangoni unstable convection originated from a droplet dissolving in a liquid/liquid system with miscibility gap, Physics of Fluids, 16(12): 4262-4272.

[16] Lappa M., (2006), Oscillatory convective structures and solutal jets originated from discrete distributions of droplets in organic alloys with a miscibility gap, Physics of Fluids, 18 (4): 042105 (14 pages). 
[17] Grant P. S., Cantor B., Rogers S. and Katgerman L., (1990), A Computer Model for Trajectories and Thermal Profiles of Atomised Droplets in Spray Forming, Cast Metals, 3(4): 227 232.

[18] Young N.O., Goldstein J.S., Block M.J., (1959), The motion of bubbles in a vertical temperature gradient, J. Fluid Mech, 6, 350-360.

[19] Subramanian R.S., (1983), "Thermocapillary migration of bubbles and droplets", Adv. Space Res., 3(5): 145-153.

[20] Balasubramaniam R. and Chai A.-T., (1987), “Thermocapillary migration of droplets: An exact solution for small Marangoni numbers”, J. Colloid Interface Sci., 119(2): 531-538.

[21] Haj-Hariri H., Nadim A. and Borhan A., (1990), "Effect of inertia on the thermocapillary velocity of a drop", J. Colloid Interface Sci., 140(1): 277-286.

[22] Nadim A., Haj-Hariri H. and Borhan A., (1990), "Thermocapillary migration of slightly deformed droplets", Part. Sci. Technol., 8(3-4): 191-198.

[23] Balasubramaniam R. and Subramanian R. S, (2000), The migration of a drop in a uniform temperature gradient at large Marangoni numbers, Phys. Fluids, 12(4): 733-743.

[24] Harper J.F. and Moore D.W., (1968), The motion of a spherical liquid drop at high Reynolds number, J. Fluid Mech., 32(2): 367-391.

[25] Haj-Hariri H., Shi Q., Borhan A., (1997), Thermocapillary motion of deformable drops at finite Reynolds and Marangoni numbers, Phys. Fluids 9 (4):845-855.

[26] Rudman M., (1998), Volume tracking methods for interfacial flow calculations, International Journal for Numerical Methods in Fluids, 24, 671-691.

[27] Gueyffier D., Li J. , Nadim A., Scardovelli S., Zaleski S., (1999), "Volume of Fluid interface tracking with smoothed surface stress methods for three-dimensional flows", J. Comput. Phys., 152: 423-456

[28] Sussman, M. and Fatemi, E. (1999): An efficient, interface-Preserving Level Set Redistancing Algorithm and its application to Interfacial Incompressible Fluid Flow. SIAM J. Sci. Comput., 20, 1165-1191.

[29] Sussman, M.; Puckett, E. (2000), A coupled level set and volume-of-fluid method for computing 3D and axisymmetric incompressible two-phase flows. J. Comput. Phys., vol. 162, pp. 301-337.

[30] Tryggvason G., Bunner B., Esmaeeli A., Juric D., Al-Rawahi N., Tauber W., Han J., Nas S., and Jan Y.-J., (2001), A Front Tracking Method for the Computations of Multiphase Flow, J. Comput. Phys., 169: 708-759.

[31] Yin Z., Gao P., Hu W. and Chang, L., (2008), Thermocapillary Migration of Nondeformable Drops, Phys. Fluid, 20, 082101.

[32] Yin Z., Chang L., Hu W., Li Q., and Wang H., (2012), Numerical simulations on thermocapillary migrations of nondeformable droplets with large Marangoni numbers, Phys. Fluids 24, 092101. 
[33] Zhao J., Zhang L., Li Z., Qin W., (2011), Topological structure evolvement of flow and temperature fields in deformable drop Marangoni migration in microgravity, International Journal of Heat and Mass Transfer, 54, 4655-4663.

[34] Brady P. T., Hermann M., Lopez J. M., (2011), Confined thermocapillary motion of a threedimensional deformable drop, Phys. Fluids, 23, 022101 (11 pages).

[35] Balcázar N., Rigola J., Castro J., Oliva A., A level-set model for thermocapillary motion of deformable fluid particles, International Journal of Heat and Fluid Flow, 2016.

[36] Liu H., Wu L., Ba Y., Xi G., A lattice Boltzmann method for axisymmetric thermocapillary flows, International Journal of Heat and Mass Transfer, (2017).

[37] Liu H. and Zhang Y., Modelling thermocapillary migration of a microfluidic droplet on a solid surface, Journal of Computational Physics, (2015).

[38] Guo Z. and Lin P., A thermodynamically consistent phase-field model for two-phase flows with thermocapillary effects, J. Fluid Mech. (2015), vol. 766, pp. 226-271.

[39] Liu H., Valocchi A. J., Phase-field based lattice Boltzmann finite difference model for simulating thermocapillary flows. Physical Review E, (2013).

[40] Liu H., Zhang Y., Valocchi A. J., Modelling and simulation of thermocapillary flows using lattice Boltzmann method, Journal of Computational Physics, (2012).

[41] Liu H., Valocchi A. J., Zhang Y., Kang Q., Lattice Boltzmann phase-field modeling of thermocapillary flows in a confined microchannel, Journal of Computational Physics (2013).

[42] Meyyappan M., Wilcox W. R., Subramanian R. S., (1981), Thermocapillary migration of a bubble normal to a plane surface, Journal of Colloid and Interface Science, 83(1), 199-208.

[43] Wozniak G., (1991), On the thermocapillary motion of droplets under reduced gravity, J. Colloid Interface Sci., 141(1): 245-254.

[44] Balasubramaniam R., Lacy C.E., Wozniak G., Subramanian R.S., (1996), Thermocapillary migration of bubbles and drops at moderate values of the Marangoni number in reduced gravity, Phys. Fluids, 8(4): 872-880.

[45] Tryggvason G., Scardovelli R., Zaleski S., (2011), Direct numerical simulations of gas-liquid multiphase flows, Cambridge University Press.

[46] Lappa M., (2005), Coalescence and non-coalescence phenomena in multi-material problems and dispersed multiphase flows: Part 2, a critical review of CFD approaches, Fluid Dynamics \& Materials Processing, 1(3): 213-234.

[47] Balasubramaniam R. and Subramanian R. S, (2001), The motion of bubbles and drops in reduced gravity, Cambridge University Press.

[48] Albadawi A., Donoghue D. B., Robinson A. J., Murray D. B., Delauré Y. M. C. (2013), Influence of surface tension implementation in volume of fluid and coupled Volume of Fluid with Level Set methods for bubble growth and detachment, Int. J. Multiphase Flows, 53, 11-28.

[49] Yamamoto T., Okano Y., Dost S., (2016), Validation of the S-CLSVOF method with the density-scaled balanced continuum surface force model in multiphase systems coupled with thermocapillary flows, Int. J. Numer. Meth. Fluids, 83(3): 223-244. 
[50] Berberović, van Hinsberg N. P., Jarkirlić S., Roisman L. V., Tropea C., (2009), Drop impact onto a liquid layer of finite thickness: Dynamics of the cavity evolution, Phys. Rev. E, 79, 036306. [51] Capobianchi P., Lappa M., Oliveira M. S. N., (2018), Implementation of a flexible and modular multiphase framework for the analysis of surface-tension driven flows based on a L-S VOF approach, in Selected papers of the 11th OpenFOAM Workshop, J.M. Nóbrega and H. Jasak (Editors), Springer Nature, 2018, ISBN - 978-3-319-60845-7.

[52] Jang D.S., Jetli R., Acharya S. (1986), Comparison of the PISO, SIMPLER and SIMPLEC algorithms for the treatment of the pressure-velocity coupling in steady flow problems. Numer. Heat Transfer 10:209-228.

[53] Moukalled F., Mangani L. and Darwish M., (2016), The Finite Volume Method in Computational Fluid Dynamics - An Advanced Introduction with OpenFOAM and Matlab, Springer International Publishing, 2016, New York).

[54] Choi SK., Nam HY., Cho M. (1994) Systematic comparison of finite-volume calculation methods with staggered and nonstaggered grid arrangements. Numer. Heat Transfer, Part B 25 (2):205-221

[55] Choi SK., Nam HY., Cho M. (1994a) Use of staggered and nonstaggered grid arrangements for incompressible flow calculations on nonorthogonal grids. Numer. Heat Transfer, Part B 25(2):193204

[56] Rhie M. C, Chow L. W., (1982), A numerical study of the turbulent flow past an isolated airfoil with trailing edge separation, AIAA-82-0998.

[57] Patankar S.V. and Spalding D.B., (1972), A calculation procedure for heat, mass and momentum transfer in three-dimensional parabolic flows, Int. J. Heat Mass Transfer, 15:1787-1806. [58] Van Doormaal J.P. and Raithby G.D. (1985), An evaluation of the segregated approach for predicting incompressible fluid flows, In National Heat Transfer Conference, Denver, Colorado, August 4-7 1985.

[59] Brackbill J.U., Kothe D.B., Zemach C., (1992), A Continuum Method for Modeling Surface Tension, J. Comput. Phys., 100 (2): 335-354.

[60] Coelho P., Pereira J.C.F., and Carvalho M.G., (1991), Calculation of laminar recirculating flows using a local non-staggered grid refinement system, Int. J. Num. Meth. Fluid, 12(6):535-557.

[61] Vilsmeier R. and HÄanel D., (1993), Adaptive methods on unstructured grids for Euler and Navier-Stokes equations, Computers and Fluids, 22(4-5):485-499.

[62] Fuster D., Bagué A., Boeck T., Le Moyne L., Leboissetier A., Popinet S., Ray P., Scardovelli R. and Zaleski S., (2009), Simulation of primary atomization with an octree adaptive mesh refinement and VOF method, International Journal of Multiphase Flow, 35, 550-565.

[63] Galusinski C. and Vigneaux P., (2008), On stability condition for bifluid flows with surface tension: application to microfluidics, J. Comput. Phys., 227 6140-64.

[64] Deshpande S. S., Anumolu L., and Trujillo M., (2012), Evaluating the performance of the twophase flow solver interFoam, Computational Science and Discovery, 5, 014016. 
[65] Ma X.J., Balasubramanian R., Subramanian R.S., Numerical simulation of thermocapillary drop motion with internal circulation, Numer. Heat Transfer A - Appl. 35 (1999) 291-239. 